\title{
High-yield Aromatic Monomers and Pure Cellulose Production by Oxidative Catalytic Fractionation of Lignocellulose in the presence of $\mathrm{CuO}$ Nanoparticles
}

\author{
Yuting Zhu, ${ }^{\mathrm{a}, \mathrm{b}}$ Yuhe Liao, ${ }^{\mathrm{a}, \mathrm{c}}$ Luying Lu, ${ }^{\mathrm{a}, \mathrm{b}}$ Wei Lv, ${ }^{\mathrm{a}}$ Jing Liu, ${ }^{\mathrm{a}, \mathrm{b}}$ Xiangbo Song, ${ }^{\mathrm{a}, \mathrm{b}}$ Jingcheng Wu, ${ }^{\mathrm{a}, \mathrm{b}}$ Xiaoping Wu, ${ }^{\mathrm{a}, \mathrm{b}}$ \\ Lei Li, ${ }^{\mathrm{d}}$ Chenguang Wang, ${ }^{*, a}$ Longlong Ma ${ }^{\mathrm{a}}$ and Bert F. Sels ${ }^{*, \mathrm{c}}$ \\ ${ }^{a}$ Key Laboratory of Renewable Energy, Guangdong Provincial Key Laboratory of New and Renewable Energy \\ Research and Development, Guangzhou Institute of Energy Conversion, Chinese Academy of Sciences, Guangzhou \\ 510640, PR China. \\ ${ }^{b}$ University of Chinese Academy of Sciences, Beijing 100049, PR China. \\ ${ }^{c}$ Center for Sustainable Catalysis and Engineering, KU Leuven, Celestijnenlaan 200f, 3001 Leuven, Belgium. \\ ${ }^{d}$ Atmospheric Environment Institute of Safety and Pollution Control, Jinan University, Guangdong 510632, PR \\ China.
}

Abstract: Thermal oxidation under mild operational conditions is a promising technology to valorize lignocellulose. However, product selectivity is restricted due to inevitable condensation of lignin fragments and degradation of monophenolics (i.e. aromatic aldehydes), while product isolation is complicated due to complete dissolution and conversion of carbohydrates (including both hemicellulose and cellulose). Herein, we report the catalytic use of multifunctional $\mathrm{CuO}$ nanoparticles (NPs) to oxidatively fractionate lignocellulosic feedstock with dioxygen in aqueous $\mathrm{NaOH}$ under mild conditions (e.g., water solvent, $160{ }^{\circ} \mathrm{C}, 1 \mathrm{MPa} \mathrm{O}_{2}$ ). In presence of $\mathrm{CuO} \mathrm{NPs,}$ lignocellulose is fractionated into three parts, overall yielding $90 \%$ carbon efficiency. Lignin is converted to up to $45.6 \mathrm{wt} \%$ in organic soluble aromatic aldehyde monomers, rich in vanillin and syringaldehyde, the value surpassing the theoretical one based on $\beta-\mathrm{O}-4$ bond content, indicating significant cleavage of other ether bonds. All hemicellulose is selectively converted into water soluble small (di)acids, mainly to oxalic acid. Up to $81 \%$ of cellulose, in contrast, is obtained as a white crystalline residue with high cellulose purity (over 95\%), which can readily be transformed into high quality nanocellulose, useful in many applications.

\section{Introduction}

In the context of global warming partially caused by usage of non-renewable fossil resources, it is of great significance but also challenging to replace fossil sources by renewable carbon for production of chemicals, fuel and materials. Lignocellulosic biomass, one type of green carbon, may have this potential. ${ }^{[1-5]}$ Utilization of cellulose and hemicellulose is widely studied and great progresses have been made both in laboratorial circumstances and at the industrial scale. ${ }^{[6-}$ 13] For instance, the Guozhen Group (China, Anhui) and Chemtex Company (China, Shanghai) are cooperating to establish a cellulosic ethanol factory with an annual production of up to 100 kilotons of ethanol from agricultural waste such as corn stalks and bagasse. GF Biochemicals Company (Italy) is planning to set up a 50,000 tons plant to directly produce levulinic acid from cellulose (C6 sugars) in 2021-2023. More applications of (hemi)cellulose are comprehensively overviewed and listed in the Tables
S12 and S13. Lignin, the other important compound of lignocellulose, is usually treated as a waste in traditional biorefineries or in the pulp and paper industry. ${ }^{[3]}$ It is key now to obtain more value from the whole lignocellulosic feedstock and thus also addedvalue should be created from lignin..$^{[3,14-23]}$

Lignin may represent a unique source of renewable aromatic chemicals, especially monophenolics. Various monophenolics have wide applications including bulk and fine chemicals, medicines, agricultural pesticides, polymers, cosmetics, food, polymers, etc. ${ }^{[16,}$ 24-31] However, it is difficult to selectively convert lignin into monophenolics because of its complex three-dimensional and amorphous structure, high recalcitrance, and their condensation upon isolation and purification. Several approaches such as oxidation, ${ }^{[32-36]}$ pyrolysis, ${ }^{[37,}{ }^{38]}$ supercritical alcohol depolymerization, ${ }^{[17,39]}$ biological treatment, ${ }^{[40]}$ mechanical treatment, ${ }^{[41]}$ organosolv treatment, ${ }^{[42]}$ are in an early developing stage to convert lignin into 
monophenolics. ${ }^{[16,17,43,44]}$ It is commonly accepted that the monophenolics yield is strongly depended on the lignin structure (thus feedstock dependent), the use and choice of (homogeneous or heterogeneous) catalysis. There is general agreement that the yield of monomers is strongly related to the presence of $\beta-O-4,{ }^{[5,45]}$ and therefore high monomer yields are better obtained from native (i.e. in planta) lignin, rather than from isolated (already condensed) lignin precipitates.

To obtain pseudo 'native' lignin, organosolv, ${ }^{[46]}$ ionic liquid, ${ }^{[47,48]}$ and deep eutectic solvent extraction (DES) $[18,49]$ are useful because of their solubilizing ability under mild reaction conditions (low temperature), but they show relatively low lignin yields. The inevitable dissolution of cellulose and hemicellulose, tedious separation steps and recovery of ionic liquid and DES remain challenging obstacles. To effectively valorize 'native' lignin, direct conversion of lignin when in the raw lignocellulosic biomass was developed. For instance, woody and grass lignocellulose were successfully converted to phenolic monomers with $\mathrm{Ni}$ $\mathrm{W}_{2} \mathrm{C} / \mathrm{AC}$ as catalyst at $235^{\circ} \mathrm{C}$ under $6 \mathrm{MPa} \mathrm{H}_{2}{ }^{\left[{ }^{[0]}\right.}$ Here, (hemi)cellulose was converted to useful compounds such as $\mathrm{C}_{2}$ to $\mathrm{C}_{4}$ diols, but their presence may complicate the monophenolics separation. Tandem fractionation of lignocellulose into its three components and further upgrading of the fractions may be the most preferred valorization option, unless a single or single type of high value product can be obtained by such full-dissolution catalytic method.

Recently, the lignin-first strategy was proposed and demonstrated to fractionate and convert lignin before valorization of the carbohydrate polymers. ${ }^{[20,21,51-53]}$ By applying this lignin-first strategy, the structurally complex lignin is converted first towards monophenolics in high yield and oligomers containing original and modified interlinkages, ${ }^{[54]}$ while carbohydrate polymers remain as an easy separable solid residue (further denoted as SR). Undesired condensation of the initially formed lignin-derived intermediates is largely inhibited by chemical stabilization or by further conversion to more stable products. To date, several lignin-first technologies were developed, such as reductive catalytic fractionation (RCF) (over $\mathrm{Ni} / \mathrm{Al}_{2} \mathrm{O}_{3},{ }^{[55]} \mathrm{Ru} / \mathrm{C},[44,56]$, $\mathrm{Zn} / \mathrm{Pd} / \mathrm{C},{ }^{[57]}$ and $\left.\mathrm{Pd} / \mathrm{C},{ }^{[58]}\right)$ oxidative catalytic fractionation (OCF) (under alkaline conditions), photocatalysis (over cadmium sulfide quantum dots) ${ }^{[59]}$ and acetal formation stabilized fractionation. ${ }^{[60,}$ 61] Compared to the above lignin-first technologies, OCF has been perhaps less practiced and investigated, although the method has clear advantages such as mild operational conditions (low temperature and pressure), green and harmless solvent $\left(\mathrm{H}_{2} \mathrm{O}\right)$ and (cheap) reagents $\left(\mathrm{O}_{2}, \mathrm{NaOH}\right)$, and most importantly, it produces unique high-value products such as aromatic aldehydes (vanillin (1G), syringaldehyde (1S), p-hydroxy benzaldehyde (1H), depending on the lignin source). Aromatic aldehydes have a wide range of applications in food, perfume, cosmetic, but they have also potential as platform chemicals in the bio-based economy for instance for bio-based polymers, $\left.{ }^{[30,} 31\right]$ pharmaceutical drugs and agricultural pesticides, provided they can be efficiently produced. ${ }^{[62,63]}$

Previous work have demonstrated that aromatic aldehydes and highly pure cellulose can be obtained by performing base-catalyzed OCF under mild stirring in absence of co-catalyst. $\left.{ }^{[32,} 33,36\right]$ However, as encountered in many wet oxidation reports and several other OCF reports, ${ }^{[32,33]}$ the yield of monophenolics was far from the theoretical yield showing a yield deficiency of more than $30 \%$. More importantly, more than $50 \%$ of high value cellulose was degraded (to carboxylic acids like formic acid, acetic acid and oxalic acid) due to its instability under the strong alkaline conditions. Moreover, monophenolics such as vanillin are very unstable in the alkaline oxidative conditions, degrading into small aliphatic acids or condensing to phenolic dimers and oligomers. ${ }^{[64]}$ The instability of those products significantly reduces (or at least complicates) the output value of the reported OCF biorefineries.

Improvement of the biomass utilization efficiency together with high mass yield for high value products during OCF is key, and therefore this work presents $\mathrm{OCF}$ in the presence of a solid additive that acts both 
as a catalyst and stabilizing reagent. Among a broad screening of oxides, $\mathrm{CuO}$ nanoparticles (NPs), preferably present as small size nanoparticles, outperforms other screened oxides. Its utilization yields monophenolics (mainly to aromatic aldehydes) with close to (or even surpass) theoretical yield (based on $\beta-\mathrm{O}-4$ bond content) and generates highly pure cellulose in up to $80 \%$ of the original cellulose weight. A modified fractionation-oxidation experiment was designed to investigate the role of $\mathrm{CuO}$ NPs on lignin fractionation, oxidation and cellulose retention. The recyclability of $\mathrm{CuO}$ NPs and the potential of the crystalline cellulose towards its transformation into high value cellulose nanocrystals were demonstrated. Overall, the presented $\mathrm{CuO}$ NPs modified OCF strategy was able to valorize different sorts of lignocellulosic feedstock with unprecedented $90 \%$ overall carbon efficiency.

\section{Oxidative catalytic fractionation over CuO NPs}

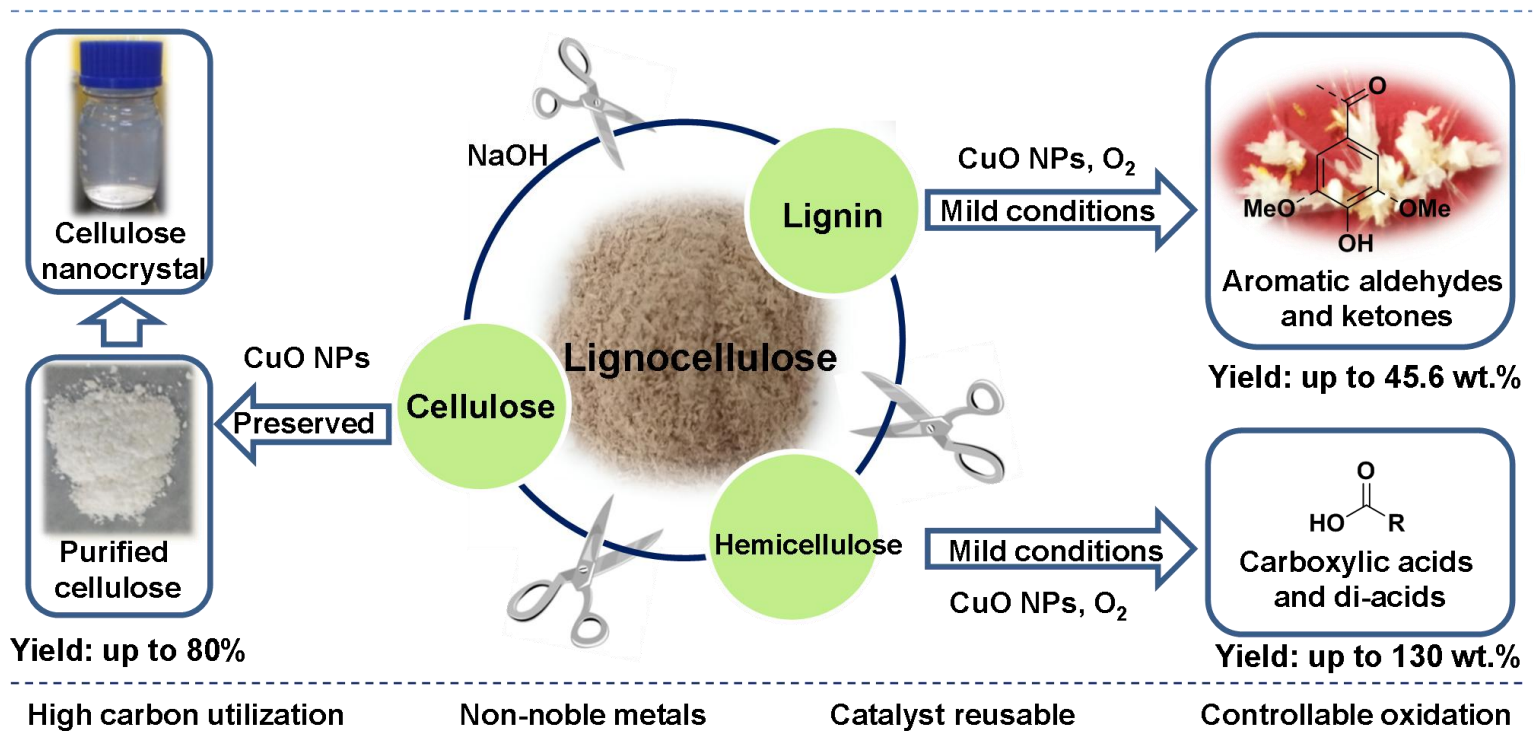

Figure 1. Oxidative catalytic fractionation (OCF) strategy for lignocellulose conversion.

\section{Results and Discussion}

\subsection{The oxidative catalytic fractionation (OCF) concept}

OCF, in analogy with RCF (reductive catalytic fractionation), is a method that simultaneously fractionates and depolymerizes lignin from lignocellulose, in the presence of $\mathrm{O}_{2}$ or air, and for safety reasons preferably carried out in water. In OCF, oxidation instead of reduction chemistry thus plays a central role. Ideally, OCF oxidizes lignin fragments into monomeric phenolic aldehydes and ketones in high yields, while hemicellulose is almost fully converted to a set of water soluble carboxylic acids, and cellulose is maximally retained as (preferably structurally intact) white crystalline solid (see Figure 1). As such, an OCF biorefinery with high carbon utilization and easy separation processes can be hypothesized, showing cellulose, monophenolics (mainly vanillin), some (solid) lignin oligomers and small acids as isolated fractions (Scheme 1). The main challenge is the hydrolysis and further oxidation of lignin with high degree of delignification and high monophenolics yield, while keeping most of the cellulose unaltered. This work therefore investigated the key role of adding selected (cheap and non-noble) solid reagents in the OCF conditions to address this challenge. To cope with the complex catalyst-solid pulp separation issues, nano-sized catalysts were studied preferably as their recovery (from the pulp) is possible by sieve fractionation, instead of using more complicated catalyst compartmentalization concepts such as catalyst basket holders, e.g. reported earlier for $\mathrm{RCF},{ }^{[55,65]}$ or magnetic nature of catalyst, like $\mathrm{Fe}-$ and Ni-based catalysts. ${ }^{[9,65,66]}$ More details of the OCF method is described in the section of Methods. 
The study starts with a broad metal oxides screening for OCF of pine wood. The best oxide is selected for a more profound parameterization, product-analytical study, and control experiments in order to get more insights in its specific role on the bio-refining of lignocellulose in OCF conditions.

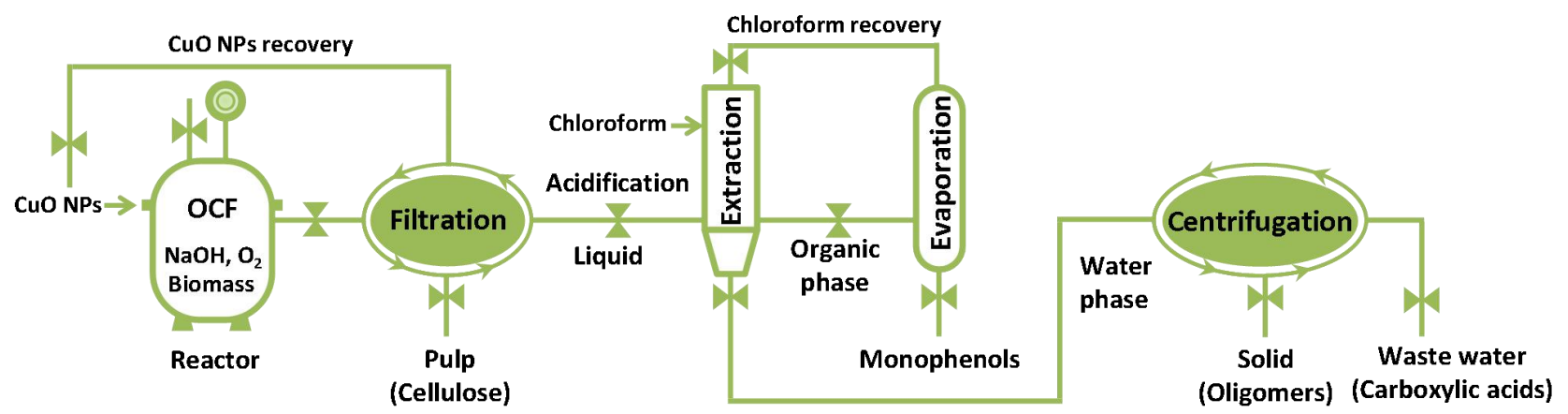

Scheme 1. Proposed process for the modified oxidative catalytic fractionation (OCF) of lignocellulose in presence of metal oxide nanoparticles, showing the different products and their isolation.

\subsection{Catalyst screening in $\mathrm{OCF}$}

Metal oxides including $\mathrm{CuO}, \mathrm{Co}_{2} \mathrm{O}_{3}, \mathrm{Co}_{3} \mathrm{O}_{4}, \mathrm{ZrO}_{2}$, $\mathrm{MoO}_{3}, \mathrm{Cr}_{2} \mathrm{O}_{3}, \mathrm{~V}_{2} \mathrm{O}_{5}, \mathrm{Fe}_{3} \mathrm{O}_{4}, \mathrm{Ta}_{2} \mathrm{O}_{5}, \mathrm{Mn}_{2} \mathrm{O}_{3}, \mathrm{TiO}_{2}$, $\mathrm{Al}_{2} \mathrm{O}_{3}, \mathrm{ZnO}$, and $\mathrm{Fe}_{2} \mathrm{O}_{3}$ were screened. Reaction conditions were set optimal in agreement with earlier reported OCF studies. ${ }^{[32,33,35,36,67]}$ The results, summarized in Figure 2, show the effect of the oxides on: (i) delignification degree including monophenolics yield and $\mathrm{Mw}$ distributions of lignin fragments (Figures 2a and S1a), (ii) hemicellulose conversion and small acids yield (Figure 2b), and (iii) solid yield including the cellulose content and purity (Figure 2c).

In agreement with the high alkalinity, the blank conditions showed high hemicellulose and lignin conversion, whereas moderate small acids $(63.2 \%)$ and monophenolics yield (26.7 wt\%) were obtained. Purity of cellulose in the solid residue is high $(89.5 \%)$, but only moderate weight of cellulose, viz. $43.5 \%$ yield, were left after pine wood OCF.

Addition of oxides shows various effects. Exceptionally, $\mathrm{CuO}$ NPs shows high $33.8 \mathrm{wt} \%$ monophenolics yields (on Klason lignin basis), which is $30 \%$ higher than without $\mathrm{CuO}$, while other oxides gave comparable $(27 \mathrm{wt} \%)$ to even lower $(<20 \mathrm{wt} \%$, such as $\mathrm{Fe}_{2} \mathrm{O}_{3}$ at similar delignification) values (Figures 2a). Its composition, comprising of vanillin as the dominant product along with acetovanillone and some $p$-hydroxy benzaldehyde, remains largely unchanged among the different oxides. Also $\mathrm{Mw}$ of the lignin fraction is impacted by the choice of oxide. Some oxides such as $\mathrm{ZrO}_{2}, \mathrm{Cr}_{2} \mathrm{O}_{3}$ and $\mathrm{Mn}_{2} \mathrm{O}_{3}$ show larger values (around $295 \mathrm{Da}$ ), whereras lower values (such as 277 and $197 \mathrm{Da}$ ) are observed for others such as $\mathrm{CuO}$ and $\mathrm{V}_{2} \mathrm{O}_{5}$, respectively (Table S1, entries 2 15 and entries 2, 5, 7, 8, 11). Although the hemicellulose conversion is fairly similar for all oxides, the small acid yield varies considerably, albeit less its composition (Figure 2b). This variation in yield is correlated oppositely to the solid retention and its cellulose content (Figure S3), indicating that cellulose is also a source of acids upon oxidation. $\mathrm{Al}_{2} \mathrm{O}_{3}$ gave the highest acid yield (93.2\%), followed by $\mathrm{Fe}_{3} \mathrm{O}_{4}$ and $\mathrm{Ta}_{2} \mathrm{O}_{5}$, whereas $\mathrm{Mn}_{2} \mathrm{O}_{3}$ shows the lowest (around $45.9 \%$ ), with the reference yield at around $60 \%$ in the absence of oxide (Figures 2b and 3). Interestingly, use of $\mathrm{CuO}$ NPs and $\mathrm{Mn}_{2} \mathrm{O}_{3}$ led to cellulose yields above $58 \%$, considerably higher than the blank. The purity of cellulose in the SRs is always high (ca. 80\%) (Figure $\mathbf{2 c}$ ), as is the crystallinity (ranging from $63.2 \%$ to $71.7 \%$ of cellulose I crystal form, Figures S7a and S7b; Table S6). The morphology of cellulose varies with the oxide used (Figure S11). SEM graphs show nanocellulosic objects with mean diameter of 19 to 28 nm (Figures S11 and S12), especially with $\mathrm{Al}_{2} \mathrm{O}_{3}$, and somewhat less with $\mathrm{Co}_{3} \mathrm{O}_{4}, \mathrm{ZrO}_{2}, \mathrm{Mn}_{2} \mathrm{O}_{3}$ and $\mathrm{TiO}_{2}$. Note that soluble species such as $\mathrm{Al}(\mathrm{OH})_{4}^{-}$are formed 


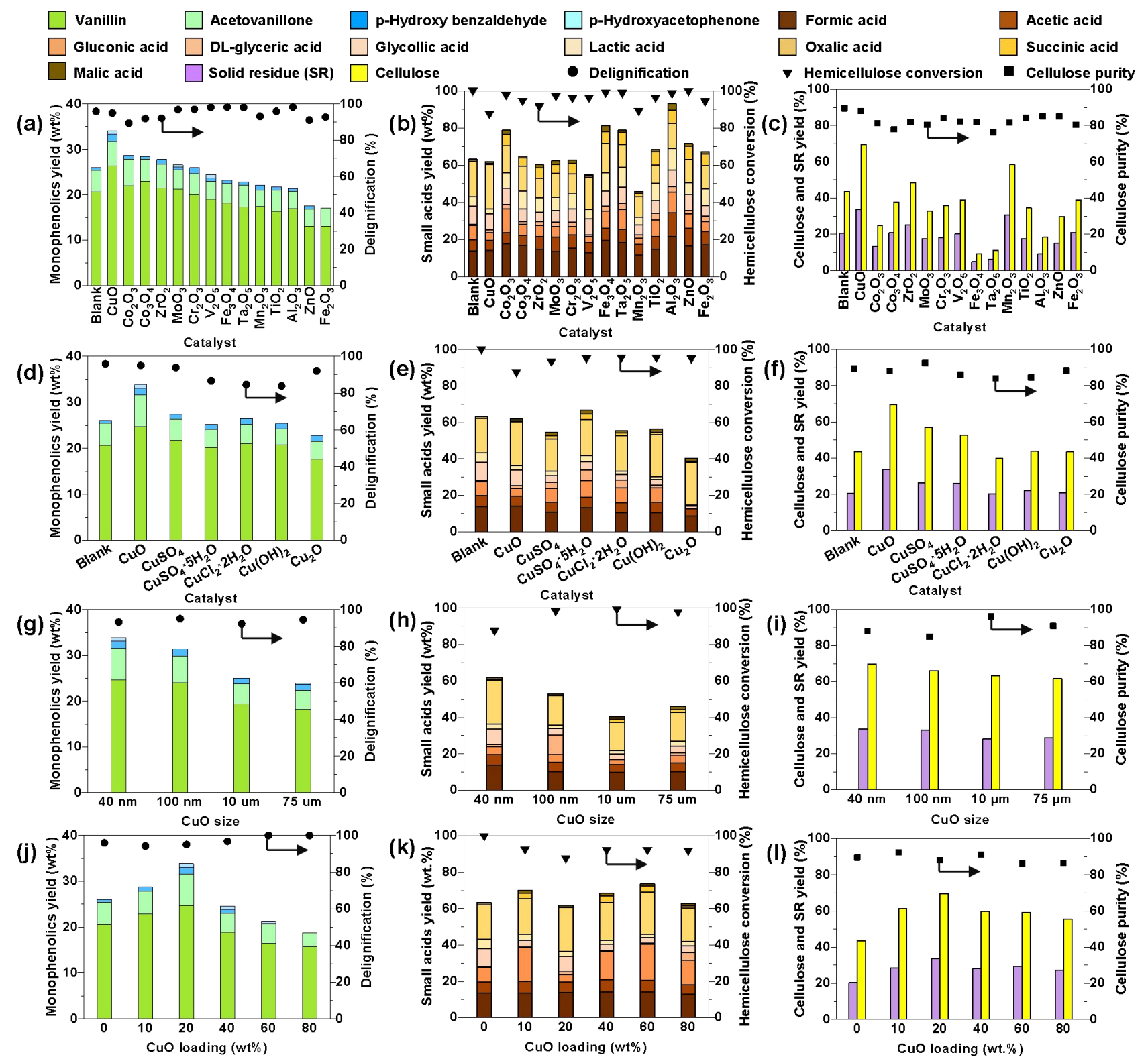

Figure 2. OCF of pine wood in the presence of metal oxides. Effect of $(a \sim c)$ metal oxides, $(d \sim f) C u$-based catalysts, ( $\sim \mathrm{i}) \mathrm{CuO}$ size, and $(\mathrm{j} \sim 1) \mathrm{CuO}$ loading on yields of monophenolics, small acids, SR and delignification, cellulose, hemicellulose conversion and cellulose purity in SR. Reaction conditions: $0.5 \mathrm{~g}$ pine, catalyst $(\mathrm{a} \sim \mathrm{c}, 1.26 \mathrm{mmol}$ metal oxides; d e, 1.26 mmol Cu-based catalyst; g h, $0.1 \mathrm{~g} \mathrm{CuO} ; \mathrm{j} \sim 1,0.1 \mathrm{~g} 40 \mathrm{~nm} \mathrm{CuO} \mathrm{NPs}), 7.5 \mathrm{wt} \% \mathrm{NaOH}$, $160^{\circ} \mathrm{C}, 60 \mathrm{~min}, 400 \mathrm{rpm}, 1 \mathrm{MPa} \mathrm{O}_{2}$.

in aqueous alkaline, potentially interacting and disturbing hydrogen bonds between the cellulose microfibrils. Cellulose formed in the presence of $\mathrm{CuO}$, $\mathrm{Co}_{2} \mathrm{O}_{3}, \mathrm{MoO}_{3}, \mathrm{Cr}_{2} \mathrm{O}_{3}, \mathrm{~V}_{2} \mathrm{O}_{5}, \mathrm{Fe}_{3} \mathrm{O}_{4}, \mathrm{Ta}_{2} \mathrm{O}_{5}, \mathrm{ZnO}$ and $\mathrm{Fe}_{2} \mathrm{O}_{3}$ have smooth intact surfaces (Figure S11).

To summarize the results, $\mathrm{CuO}$ NPs behave superior to the other oxides in alkaline OCF of pine wood, showing high (33.8 wt\%) monophenolics (with low $\mathrm{Mw}$ of lignin fragments) and $69.6 \%$ cellulose yield, corresponding to $88.1 \%$ of cellulose (type I) purity (with smooth cellulose surfaces) in SR, with a moderate small acid yield, at (almost) complete lignin and hemicellulose conversion. The yields of aromatic monomers and cellulose are improved $30 \%$ and 58\%, respectively, compared to without oxides.

With these promising OCF results in hand, we tested other common $\mathrm{Cu}$-based salts, oxides and hydroxides such as $\mathrm{CuCl}_{2}, \mathrm{CuSO}_{4}, \mathrm{Cu}(\mathrm{OH})_{2}$ and $\mathrm{Cu}_{2} \mathrm{O}$. Pourbaix 
diagrams suggest that $\mathrm{Cu}$ is favorably present as $\mathrm{Cu}(\mathrm{II}) \mathrm{O}$ at high $\mathrm{pH}$ and metal potential (high oxygen content). ${ }^{[68]}$ Although one might expect similar results for all, differences may still be encountered due to different kinetics and complicated particle (size) formation under reaction conditions en route to $\mathrm{CuO}$. Indeed, the results of $\mathrm{CuO} \mathrm{NPs}$ with regard to monophenolics and cellulose yield are better than those of all other $\mathrm{Cu}$ precursors (Figure 2d-f). Remarkably, the cellulose after OCF treatment over $\mathrm{CuO}$ NPs is in cellulose I crystal form, whereas use of $\mathrm{Cu}_{2} \mathrm{O}$ transforms cellulose I into cellulose II crystals (Figure S7c). Accordingly, the surface is smooth for $\mathrm{CuO}$, but rather rough for $\mathrm{Cu}_{2} \mathrm{O}$ due to the crystal transformation, as confirmed by SEM (Figures S13 and S14; Text S1).

The particle size of $\mathrm{CuO}$ appears an important factor. This became clear after testing a range of commercial $\mathrm{CuO}$ with particle sizes ranging from 0.04 to $75 \mu \mathrm{m}$. The OCF results are summarized in Figure 2g-i. Most apparent trend is the high monophenolics (with particularly increase of vanillin and acetovanillone) and cellulose yield for the smallest $40 \mathrm{~nm}$ particle size, whereas the changes of all other OCF performances (cellulose crystal type, crystallinity and surface roughness, small acids yield, hemicellulose and lignin conversion) are less significant (Figures 2g-i, S7d and S15).

Use of different $40 \mathrm{~nm} \mathrm{CuO} \mathrm{NPs} \mathrm{loadings} \mathrm{also} \mathrm{affects}$ pine OCF results (Figure 2j-l, S1s and S16). Use of $20 \mathrm{wt} \%$ (compared to the lignocellulose mass) appears the most optimal oxide loading under the applied OCF conditions, as substantially higher monophenolics and cellulose yields were obtained with this $\mathrm{CuO}$ NPs loading.

\subsection{Impact of the traditional OCF parameters on the CuO NPs system}

The impact of other OCF conditions such as temperature, base concentration, stirring rate and reaction time is systematically investigated for the 40 $\mathrm{nm} \mathrm{CuO} \mathrm{NPs} \mathrm{(20} \mathrm{wt \%} \mathrm{loading)} \mathrm{system.} \mathrm{The} \mathrm{results} \mathrm{are}$ summarized in Figure 3.

Clearly, CuO NPs modified OCF also needs sufficient amount of base (Figure 3a-c). This is consistent with other OCF studies. ${ }^{[32,36]}$ Higher base concentration increases both lignin and hemicellulose conversion, but decreases the cellulose yield (i.e. more loss of cellulose albeit that the obtained cellulose is more pure). With regard to the monophenolics yield, use of $7.5 \mathrm{wt} \%$ $\mathrm{NaOH}$ is optimal, which is also optimal to other oxidefree OCF processes, but the latter show lower monophenolics yield, usually well below $20 \mathrm{wt} \%{ }^{[35,69]}$

A temperature of $160^{\circ} \mathrm{C}$ is optimal for monophenolics and cellulose yield, and the cellulose purity (Figure 3d-f). Lower temperatures cause incomplete lignin and hemicellulose conversion, whereas higher temperature causes oxidation of cellulose and lignin (and monophenolics) into small acids, showing a clear increase in oxalic acid content. The increase of oxalic acid is mostly attributed to oxidation of cellulose (Figure 3e-f), as it has been reported through a dominant $\mathrm{C} 2$ retro-aldol pathway in previous research. ${ }^{[70]}$ A notable $79 \mathrm{wt} \%$ of oxalic acid, an attractive end-product by itself with wide applications in bleaching, cleaning, metallurgical industry, pharmaceutical industry, dyeing and printing industry, organic synthesis and cosmetics industry, ${ }^{[71,72]}$ was thus obtained at $200^{\circ} \mathrm{C}$, but this is not the focus of this work.

The crystallinity of cellulose, remaining in cellulose crystal I form, increases from $54.3 \%$ to $72.9 \%$, with the temperature, showing crystals with smooth surfaces (Figures S7g and S18a-d, Table S6), until a crystal transformation to cellulose crystal form II and clear roughening of the surface sets in at $200^{\circ} \mathrm{C}$ (Figure S18e). 

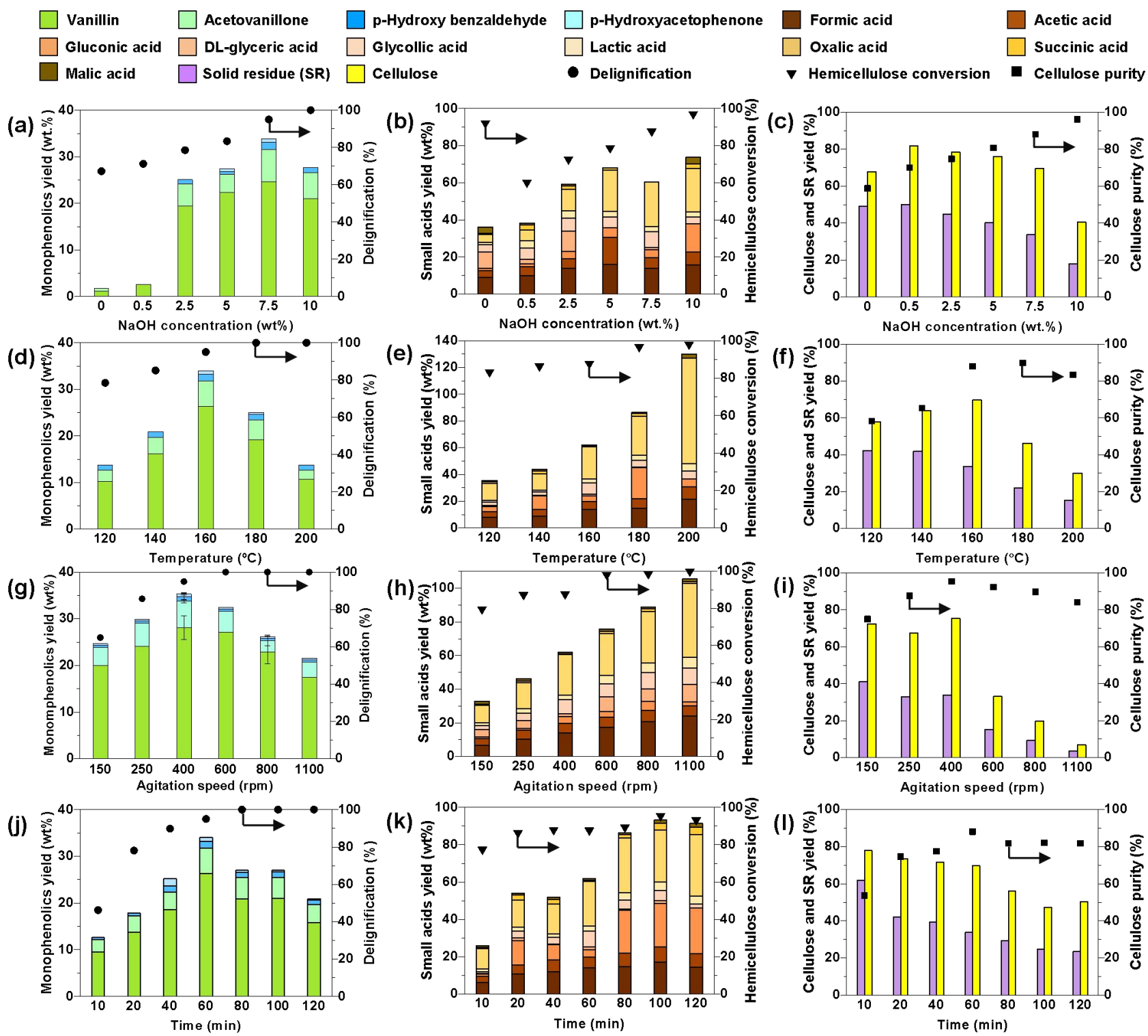

Figure 3. OCF of pine wood at different reaction conditions in the presence of $\mathrm{CuO}$ NPs. Effect of $(\mathrm{a} \sim \mathrm{c}) \mathrm{NaOH}$ concentration, $(\mathrm{d} \sim \mathrm{f})$ reaction temperature, $(\mathrm{g} \sim \mathrm{i})$ agitation speed and $(\mathrm{j} \sim \mathrm{l})$ reaction time on yields of monophenolics, small acids, SR and cellulose, delignification, hemicellulose conversion and cellulose purity in SR. Reaction conditions: $0.5 \mathrm{~g}$ pine, $0.1 \mathrm{~g} 40 \mathrm{~nm} \mathrm{CuO} \mathrm{NPs,} 1 \mathrm{MPa} \mathrm{O}_{2}$, (a c ): 0.5 10 wt $\% \mathrm{NaOH}, 160^{\circ} \mathrm{C}, 60 \mathrm{~min}, 400 \mathrm{rpm}$; (d f): $7.5 \mathrm{wt} \% \mathrm{NaOH}, 120 \sim 200^{\circ} \mathrm{C}, 60 \mathrm{~min}, 400 \mathrm{rpm}$; (g i): $7.5 \mathrm{wt} \% \mathrm{NaOH}, 160^{\circ} \mathrm{C}, 60 \mathrm{~min}, 150 \sim 1100 \mathrm{rpm}$; (j 1): 7.5 wt $\% \mathrm{NaOH}, 160^{\circ} \mathrm{C}, 10 \sim 120 \mathrm{~min}, 400 \mathrm{rpm}$.

OCF with $40 \mathrm{~nm} \mathrm{CuO} \mathrm{NPs} \mathrm{requires} \mathrm{a} \mathrm{stirring} \mathrm{rate} \mathrm{of}$ $400 \mathrm{rpm}$ to achieve the best results in terms of monophenolics and cellulose yield and the cellulose purity (Figure 3g-i). Lower stirring speed inhibits conversion of lignin and hemicellulose, whereas vigorous stirring substantially lowers the cellulose yield in favor of mainly oxalic acid formation, a phenomenon that corroborates our earlier observation on the impact of stirring rate in oxide-free OCF. ${ }^{[36]}$
Similarly as with temperature, lower monophenolics and cellulose yields coincide with higher small acids (mainly oxalate) yield. Cellulose is fairly crystalline under the different stirring regimes (Figure S7h), until vigorous stirring clearly breaks the cellulose structure, making it more amenable for conversion by oxidation to (mainly oxalic) acid (Figures S19 and $4 \mathrm{~h}$ ).

Finally, the reaction time was varied for the above optimal conditions, viz. $160^{\circ} \mathrm{C}, 7.5 \mathrm{wt} \% \mathrm{NaOH}$ and 
$400 \mathrm{rpm}$ (Figure 3j-1). Whereas the hemicellulose conversion was already high in the early time stage of the reaction, OCF requires sufficient time, viz. at least $60 \mathrm{~min}$, to establish complete lignin conversion. Longer reaction times increase the small acid yields at the expense of solid cellulose. Particularly, oxalic acid and gluconic acid are formed. Due to the lower temperature, carbon-carbon cleavage of the retro-aldol reaction is retarded thus favoring the direct glucose oxidation to gluconic acid. Cellulose purity was highest after a reaction time of $60 \mathrm{~min}$ or longer. Inspection of the crystals with SEM shows that the sheet-shaped structures on the surface (Figure S20a and S24a) typically for lignin, disappear with time until cellulose crystals (cellulose I, according to XRD) appear with smooth surfaces (Figure S20b-g). This evolution is in line with the gradual delignification in time until completeness above $60 \mathrm{~min}$.

There are lignin fragments other than the monophenolics present in the solution after OCF, which were so far not described. Although their individual chemical structures are more complex, and not part of this study, analyzing their Mw and PD may already help clarifying the underlying chemistry of the alkaline OCF in presence of CuO NPs. Figures S1 and S2 showed the molecular weight distribution of products in the organic phase. Clearly, the intense elution peak at ca. $150 \mathrm{~g} / \mathrm{mol}$ corresponded to a typical molecular weight of monophenolics. The next two elution signals, at 240 and $300 \mathrm{~g} / \mathrm{mol}$, corresponds to C-C linked dimers (Figures S35-S37). The rest of the elution at the higher molecular weight range refer to the larger phenolic oligomers. Figure 4 describes the evolution of the molecular weight ( $\mathrm{Mw}$ and $\mathrm{PD}$ ) of these lignin fragments in relation to the different OCF operational conditions. Both $\mathrm{NaOH}$ and temperature show a U-shaped relationship with $\mathrm{Mw}$ and $\mathrm{PD}$ (Figures 4a-b, S2a-b; Table S2, entries 1 11). The high $\mathrm{Mw}$ and $\mathrm{PD}$ at low values correspond to a restricted lignin conversion under mild OCF conditions, whereas the high values at the severest conditions likely coincides with the formation of condensed products or the forced dissolution of some larger and more resistant lignin fragments. Highest Mw (600 Da) and PD (around 2.5) were observed at low and high temperature, whereas differences in $\mathrm{Mw}$ and $\mathrm{PD}$ are less significant upon varying the $\mathrm{NaOH}$ concentrations. The high PDs indicate that more than one compound is formed with small difference in $\mathrm{Mw}$. In the optimal conditions, at $160^{\circ} \mathrm{C}$ and $7.5 \mathrm{wt} \% \mathrm{NaOH}$, an $\mathrm{Mw}$ around $250 \mathrm{Da}$ with very low PD (around one) were observed. Agitation and contact time show opposite trends with regard to the evolution of $M \mathrm{w}$ and $\mathrm{PD}$ (Figure 4c-d, Table S2, entries 12-24). Higher agitation rate shows a decrease in $\mathrm{Mw}$ and $\mathrm{PD}$, whereas higher stirring values for these structural parameters were found with increasing contact time.

In line with our goal to achieve co-production of monophenolics and cellulose, we have selected the optimal reaction conditions, i.e. OCF of pine wood requires $7.5 \mathrm{wt} \% \mathrm{NaOH}$ and $160^{\circ} \mathrm{C}$ for a reaction time of one hour under $400 \mathrm{rpm}$ stirring. These conditions were used for further study.
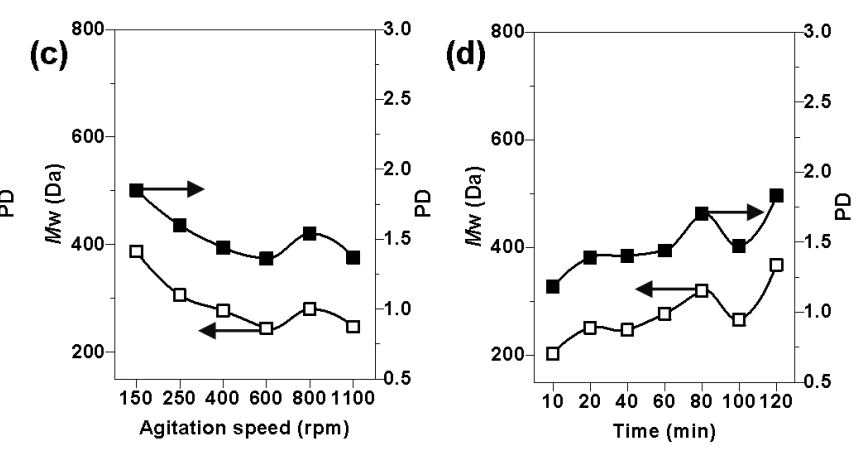

Figure 4. Weight-average molecular weight $(M \mathrm{w})$ and polydispersity $(\mathrm{PD})$ of lignin fragments under different (a) $\mathrm{NaOH}$ concentrations, (b) reaction temperature, (c) agitation speed and (d) reaction time. $\mathrm{PD}=M \mathrm{w} / M \mathrm{n}$. 


\subsection{Role of $\mathrm{CuO}$ NPs in the conversion of lignin}

This part attempts to reveal the role of $\mathrm{CuO}$ NPs and dioxygen in OCF of pine wood. For that, a set of experiments attempting a disentanglement of lignin depolymerisation and oxidation were designed. The individual effects of $\mathrm{CuO}$ NPs and dioxygen on lignin conversion with regard to delignification, monophenolic yield and $\mathrm{Mw}$ of soluble lignin fragments were therefore investigated systematically. The results are summarized in Figure 5.

A first experiment (Method I, step 1) fractionates pine wood in alkaline solution in the absence of $\mathrm{CuO}$ NPs and dioxygen (under otherwise optimal conditions: 7.5 wt $\% \mathrm{NaOH}, 400 \mathrm{rpm}, 160^{\circ} \mathrm{C}$ and $\left.60 \mathrm{~min}\right)$. Delignification was high (73\%) but incomplete (Figures S25a and S39a). It resulted in the cleavage of ether bonds between cellulose/hemicellulose and the lignin, and partly of the ether bonds in substructure A ( $\beta-\mathrm{O}-4)$, B ( $\left.\alpha-\mathrm{O}-\gamma^{\prime}\right)$, and $\mathrm{C}(\alpha-\mathrm{O}-4)$ within lignin, as evidenced by the appearance of weak signals of $\mathrm{A}, \mathrm{B}$ and $\mathrm{C}$ and products of ether bond cleavage in the HSQC spectra (Figures S43-S47, Text S4). Some monophenolics (with some vanillin and acetovanillone) along with 5-5 linked dimers can be formed from cleavage of all the ether bonds in $\mathrm{F}(\alpha-\mathrm{O}-4 / \beta-\mathrm{O}-4)$ (Figures S36, S38 and S43; Text S4). In addition, vanillin and acetovanillone can be formed from cleavage of ether bond in substructure $\mathrm{A}, \mathrm{B}$, and $\mathrm{C}$ (Scheme 2a, Figures S36 and S38). Over 70\% of hemicellulose and a small amount of cellulose were converted, forming significant amounts of small acids, mainly glyceric acid (Figure S25b), likely due to oxido-reduction pathways involving hydride shifts with the initial retro-aldol products under alkaline conditions (Figure S26b; Text S2). The soluble fraction contains a large part of unknowns, corresponding to $20.4 \%$ of carbon, next to acidinsoluble lignin oligomers, small acids and very little monophenolics (Figures S4, S25a-b and S36). FT-IR analysis suggests the unknown products are acidsoluble lignin and aliphatic acids (likely from extractives) (Figure S41).
Subsequent oxidation of the soluble fraction with dioxygen (in absence of CuO NPs; (Method 1, step 2), appears to convert some of the acid insoluble lignin fraction and acid-soluble lignin (soluble in the $\mathrm{HCl}$ aqueous solution) with clear formation of monophenolics (predominantly vanillin and acetovanillone), while a substantial addition of small acids is apparent (most likely products from the aliphatic acids) (Figures 5a, S25a-b).

When adding $\mathrm{CuO}$ NPs in step 2 (i.e. method 1, step 2a, Figure 5a), considerably more monophenolics were formed by converting more acid-insoluble lignin fraction and acid-soluble lignin. This proves that the presence of $\mathrm{CuO}$ NPs is essential to facilitate the selective oxidation of the lignin fraction towards monophenolics. Vanillin is the major compound, next to acetovanillin, some $p$-hydroxy benzaldehyde and $p$ hydroxy acetophenone. A larger amount of oxalic acid was also obtained, likely through a $\mathrm{Cu}$-assisted oxidation of intermediate acids such as glycolic acid and lactic acid. ${ }^{[70,73]}$ The acids contain more gluconic acid, likely due to oxidation of glucose assisted by $\mathrm{CuO}$ NPs (see Figure S25b).

A second series of experiments starts with delignification in the presence of CuO NPs, but in the absence of dioxygen (Method II, step 1; Figure 5b). In the presence of $\mathrm{CuO} N \mathrm{NP}$, more lignin is converted showing 89\% delignification (Figure S25a and S36), yet its conversion remains below that for a reaction in in the presence of $\mathrm{O}_{2}$ or both $\mathrm{CuO}$ NPs and $\mathrm{O}_{2}$ (Methods III and VI, Figure 5c-d). The conversion of cellulose remains insignificant, indicating $\mathrm{CuO}$ NPs fails to convert cellulose, as evidenced by its abundant presence in the FT-IR spectra of the solid residue (Figure S39). Interestingly, presence of $\mathrm{CuO}$ NPs doubled the amount of small acids at the expense of unknowns (aliphatic acid and acid soluble lignin), while more oxalic acid is formed at the cost of gluconic acid. Less amount of acid-insoluble lignin fraction was obtained compared to the OCF reaction without $\mathrm{CuO}$. Correspondingly, more monophenolics and 5-5 linked dimers (instead of oligomers) were formed in presence of $\mathrm{CuO}$ NPs (Figure S36). $\mathrm{CuO}$ 
NPs can act as oxidant to oxidize $\mathrm{C}_{\alpha}=\mathrm{C}_{\beta}$ side chain of lignin (fragments) to vanillin or oxidize $\mathrm{C}_{\gamma}-\mathrm{OH}$ of lignin containing $\mathrm{C}_{\alpha}-\mathrm{OH}$ and $\mathrm{C}_{\gamma}-\mathrm{OH}$ in the aliphatic side chain to vanillin (Scheme $2 \mathrm{~b}$; Figure S38a, route iii and iv; Figure S38b-d; Text S4). The dark purple color of spent catalyst suggests that $\mathrm{CuO}$ NPs was partially reduced. XRD analysis indeed showed the pattern of $\mathrm{Cu}_{2} \mathrm{O}$ and $\mathrm{Cu}$ instead of the initial $\mathrm{CuO}$ Figure S8a). Then, contacting the soluble fraction with dioxygen without $\mathrm{CuO}$ NPs (Method II, step 2) increases the yield of monophenolics, small acids and acid-soluble lignin fraction, while acid-insoluble lignin fraction is substantially decreased. The same step but in the presence of CuO NPs (Method II, step 2a) even further increases the small acids part and the acidsoluble lignin fraction with further decreasing of the acid-insoluble fraction, whereas the yield of monophenolics was decreased likely due to their overoxidation.

The above observations with regard to the role of $\mathrm{O}_{2}$ and $\mathrm{CuO}$ NPs suggest that: i) alkaline condition is required to solubilize lignin by cleavage of ether bonds (Figures 5a, S4 and S36; Table S3, entries 1-6; Scheme 2a); ii) $\mathrm{CuO}$ NPs itself is able to oxidatively cleave the bonds of lignin, likely through cleavage of epoxide or a redox mechanism forming reduced $\mathrm{Cu}$ species (Scheme 2b; Figures S8a, S31, S36, S39, S43-S46; Text S4) in line with earlier reports, ${ }^{[74,75]}$ but the presence of both dioxygen and $\mathrm{CuO}$ breaks interlinkages of lignin more efficient to form vanillin, for which a $\mathrm{CuO}$ NPs assisted pathway through a free radical mechanism is suggested in Scheme $2 \mathbf{c}$ and Text S3; iii) CuO NPs in the presence of dioxygen

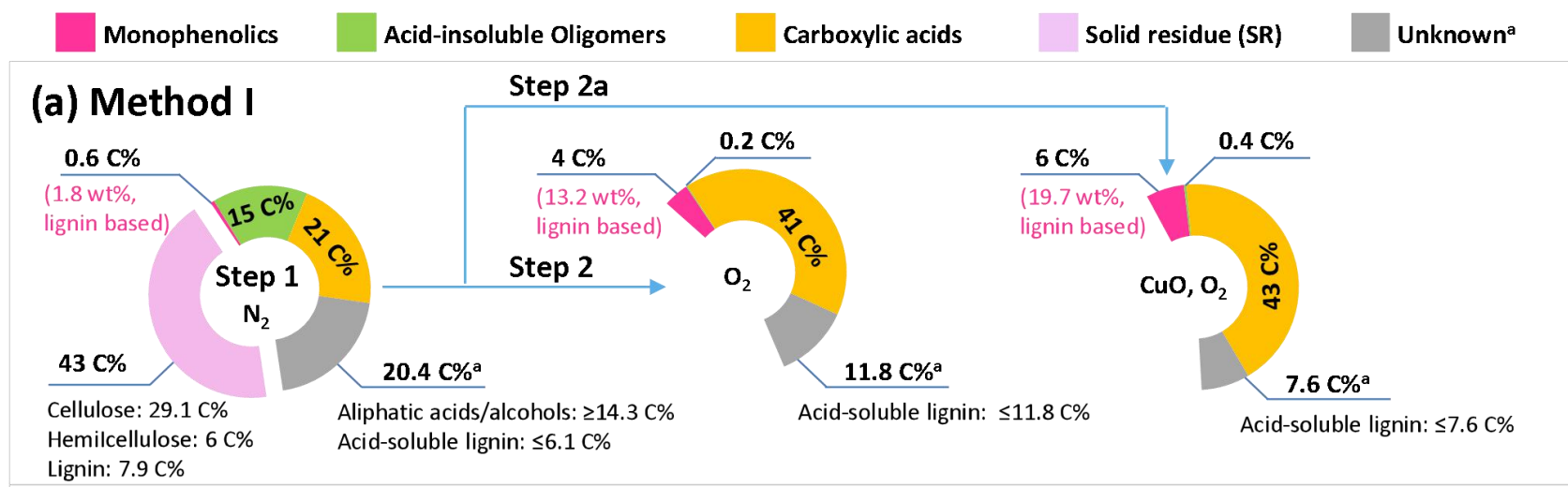

\section{(b) Method II}

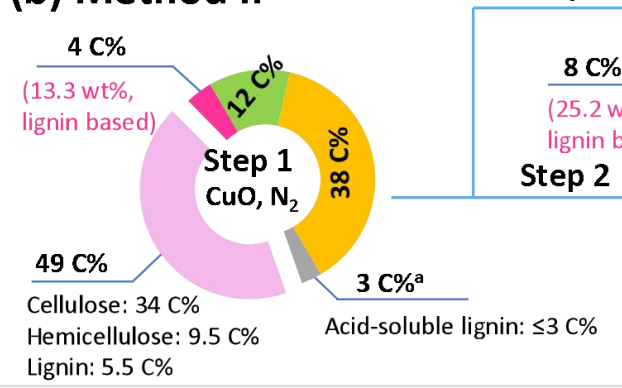

\section{(c) Method III}
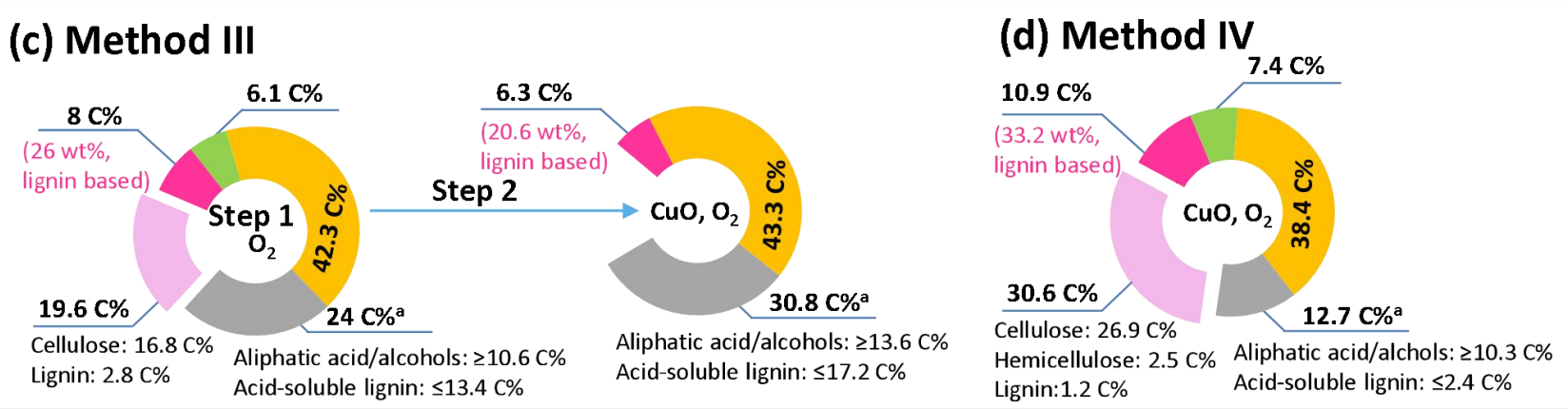
Figure 5. Effects of $\mathrm{CuO}$ NPs on lignocellulose fractionation and oxidation. Method I: (step 1) fractionation of pine under $\mathrm{N}_{2}$ followed by (step 2) oxidation under $\mathrm{O}_{2}$ in the absence or (step 2a) presence of CuO NPs. Method II: (step 1) fractionation of pine under $\mathrm{N}_{2}$ in the presence of $\mathrm{CuO}$ NPs followed by (step 2) oxidation under $\mathrm{O}_{2}$ in the absence or (step 2a) presence of $\mathrm{CuO}$ NPs. Method III: (step 1) fractionation of pine under $\mathrm{O}_{2}$ followed by (step 2) oxidation under $\mathrm{O}_{2}$ in the presence of $\mathrm{CuO}$ NPs. Method VI: OCF of pine $\mathrm{O}_{2}$ in the presence of CuO NPs. All carbon yields are on the basis of biomass carbon molar. Carbon yield of oligomers is on the assumption that all lignin units are guaiacyl $(\mathrm{G}, \mathrm{Mw}=196 \mathrm{Da})^{[21]}$ since pine lignin is mostly $\mathrm{G}$ lignin (Figure $6 \mathrm{~g}$ ). ${ }^{\mathrm{a}}$ The unknown products includes acid soluble lignin and aliphatic acids according to FTIR results (Figure S41). Note: the acid soluble lignin refers to the acid soluble lignin fraction after $\mathrm{HCl}$ acidification of alkaline fractionated lignin. Yield of unknown products (gray portion) is equal to the $100 \mathrm{C} \%$ minus the yields of solid residue (lilac portion), monophenolics (violet portion) and oligomers (green portion) and carboxylic acids (yellow portion). Reaction conditions: (step 1) $0.5 \mathrm{~g}$ pine, $7.5 \mathrm{wt} . \% \mathrm{NaOH}, 160^{\circ} \mathrm{C}, 60 \mathrm{~min}, 1 \mathrm{MPa} \mathrm{N} \mathrm{N}_{2}$ or $\mathrm{O}_{2}, 400 \mathrm{rpm}, \mathrm{CuO}$ NPs $(0 \mathrm{~g}$ or $0.1 \mathrm{~g}$ ); (step 2) alkaline solution from step 1, $1 \mathrm{MPa} \mathrm{O}_{2}, 400 \mathrm{rpm}, 160^{\circ} \mathrm{C}, 60 \mathrm{~min}$; (step 2a) alkaline solution from step 1, $0.1 \mathrm{~g} \mathrm{CuO} \mathrm{NPs}, 1 \mathrm{MPa} \mathrm{O}, 400 \mathrm{rpm}, 160^{\circ} \mathrm{C}, 60 \mathrm{~min}$.

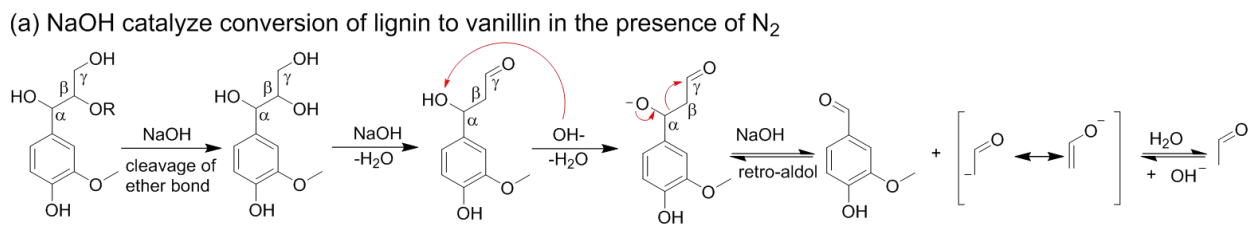

(b) $\mathrm{CuO}$ catalyze oxidation of lignin to vanillin in the presence of $\mathrm{N}_{2} \quad \mathrm{R}=\mathrm{H}$ or lignin units (S/G/H units)

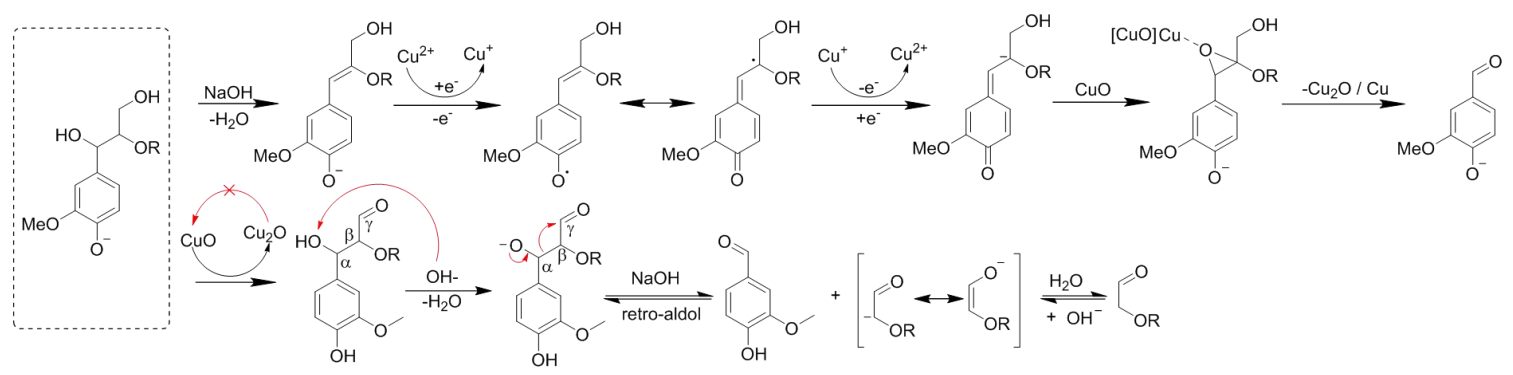

(c) $\mathrm{CuO}$ catalyze oxidation of lignin to vanillin in the presence of $\mathrm{CuO}$ and $\mathrm{O}_{2} \quad \mathrm{R}=\mathrm{H}$ or lignin units (S/G/H units)

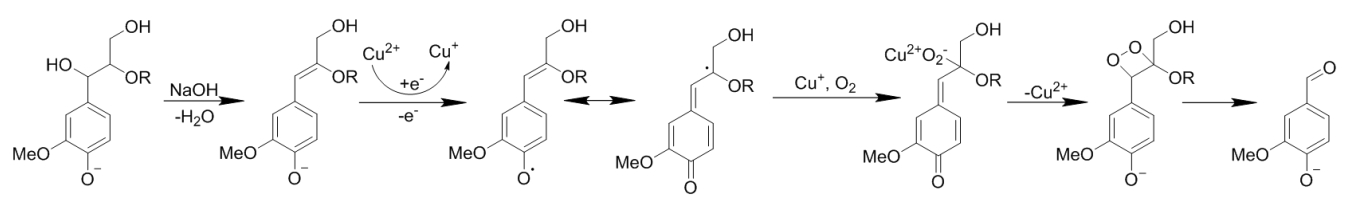

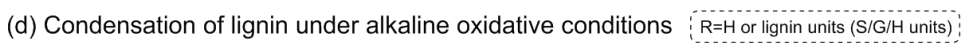

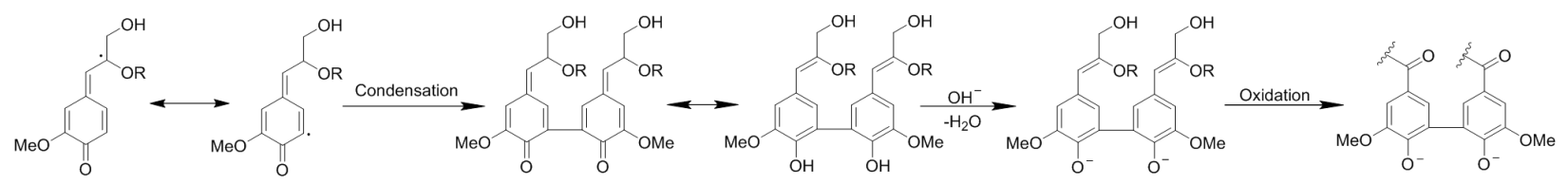

Scheme 2. ${ }^{[76]}$ Possible pathways of conversion of lignin to vanillin and (d) condensation of lignin to 5-5 linked dimers.

is also effective in oxidation of polysaccharides, while competitive pathways through retro-aldol chemistry to form oxalic acid may be predominant depending on temperature and $\mathrm{pH}$ (Figures 4 and S34); and iv) stability of monophenolics, especially in the presence of $\mathrm{CuO}$ NPs and $\mathrm{O}_{2}$, is limited as aldehydes and ketones are prone to further oxidize into acids or others. ${ }^{[28,77]}$ 
In order to further investigate the roles of $\mathrm{CuO} \mathrm{NPs}$ on the lignin conversion, HSQC NMR spectroscopy was conducted on the chloroform extracted lignin fragments obtained from OCF of pine in presence and absence of $\mathrm{CuO}$ NPs, and compared to the results of a reference, pine milled wood lignin (MWL). The results are presented in Figure 6.

Inspection of aliphatic end groups (Figure 6a-c) shows disappearance of methyl $(\delta \mathrm{C} / \delta \mathrm{H}=15 / 1 \mathrm{ppm})$ and $20.5 / 2$ ppm and 25/1.5 ppm), and appearance of the signal of the methylketone (e.g., of acetovanillone and phydroxyacetophenone units).

Inspection of the interlinkage region $(\delta \mathrm{C} / \delta \mathrm{H}=45 \sim 90 / 2.5 \sim 6 \mathrm{ppm})$ shows all original substructures including A, A' $(\beta-\mathrm{O}-4), \mathrm{B}(\alpha-\mathrm{O}-\gamma / \beta-\beta)$, $\mathrm{C}(\beta-5 / \alpha-\mathrm{O}-4), \mathrm{E}(\beta-4)$ and $\mathrm{F}(5-5 / \alpha-\mathrm{O}-4 / \beta-\mathrm{O}-4)$, irrespective of the presence (or not) of $\mathrm{CuO}$ NPs (Figure 6e-f; Table S10). The combined results of GC-MS (Figures S35-S37) and HSQC thus suggest that original substructures were converted, and they follow base-catalyzed hydrolysis mechanisms for ether bond interlinkage cleavage, followed by dehydration/oxidation steps (Scheme 2a; Figures S38;

Text S4). More interestingly, a few new signals at $\delta \mathrm{C} / \delta \mathrm{H}=62 / 4.5 \mathrm{ppm}$ were found, assigned to the $\mathrm{C}_{\gamma}-\mathrm{OH}$ in the diketone substructure (M). The $\mathrm{M}$ can be formed via oxidation of $\mathrm{C}_{\alpha}-\mathrm{OH}$ and $\mathrm{C}_{\beta}-\mathrm{OH}$ in substructure $\mathrm{P}$ (main product of cleavage of $\beta-\mathrm{O}-4$ bond, Figure $\mathbf{S 4 3}$ ) by $\mathrm{O}_{2}$ and $\mathrm{CuO} N P s$, and realizing a high stability due to conjugation in the molecular structure. Less $\mathbf{M}$ exists in the presence of $\mathrm{CuO}$ NPs because $\mathrm{CuO}$ NPs catalyse lignin to benzaldehydes. In addition, signal at $51.34 / 3.55$ ppm assigned to $\mathrm{C}_{\beta}-\mathrm{H}_{\beta}$ in substructure $\mathbf{N}$ in the presence of $\mathrm{CuO}$ NPs. The $\mathbf{N}$ probably generated by oxidation of $\mathrm{C}_{\alpha}-\mathrm{OH}$ in $\mathbf{Q}$, followed by dehydration of $\mathrm{C}_{\beta}-\mathrm{OH}$ (Figure 6e-f). Therefore, the interlinkages of the oligomers are probably $\mathrm{C}-\mathrm{C}$ (aliphatic or $\mathrm{C}_{\text {aryl }}-\mathrm{C}_{\text {aryl }}$ like 5-5) bonds.

The aromatic region showing dominant signals of $\mathrm{C}_{\alpha-}$ keto-guaiacyl units (in G', G', and G',') together with $\mathrm{C}_{\alpha}$-keto-p-hydroxyphenyl (in $\mathrm{H}, \mathrm{H}^{\prime}$, and $\mathrm{H}^{\prime \prime}$ ) analogues at the expense of $\mathrm{G}$ and $\mathrm{H}$, reveals the effective oxidation. Their intensity is highest in the presence of $\mathrm{CuO}$ NPs corroborating the high oxidation ability by combining $\mathrm{CuO}$ NPs with dioxygen. Crosslinking signals of the vanillin end group are clearly present at 190.9/9.8 ppm (Figure 6g-i; Table S10). The HSQC assignments thus corroborate the presence of monophenolics $\mathbf{1 G}, \mathbf{2 G}, \mathbf{1 H}$ and $\mathbf{2 H}$, but also dimer substructures such as dehydrodivanillin (G'), dehydrovanillin ethyl ketone (G'") were found, and significantly more 5-5 compounds are present in the absence of CuO NPs (Table S10). These substructures G" and G", can be formed by condensation chemistry (homolytically and heterolytically) of vanillin, ${ }^{[78]}$ acetovanillone (Scheme 2d), or by cleavage of the ether bond and end group oxidation of dibenzodioxocin substructures (F) (Figure 6h-i). Notable, the amount of $G$ " and $G$ "' is similar to that of $F$ in the presence of $\mathrm{CuO}$ NPs, while it is twice of $\mathrm{F}$ in the absence of $\mathrm{CuO}$ NPs (Table S10). This result suggests that $\mathrm{CuO}$ NPs facilitates the rapid formation of vanillin and acetovanillone, disfavoring the (slower) condensation chemistry at the optimize conditions (Scheme 2d). In addition, all cinnamyl aldehyde (J) units were all converted. 
(a)

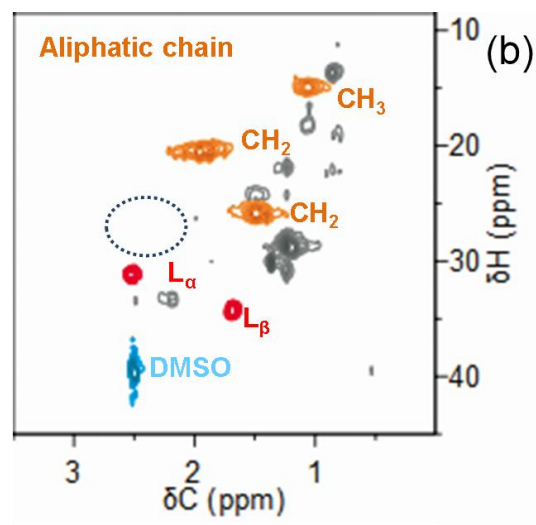

(d)

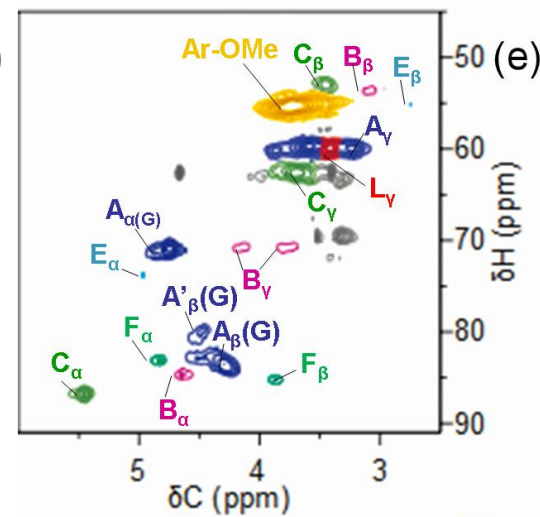

(g)

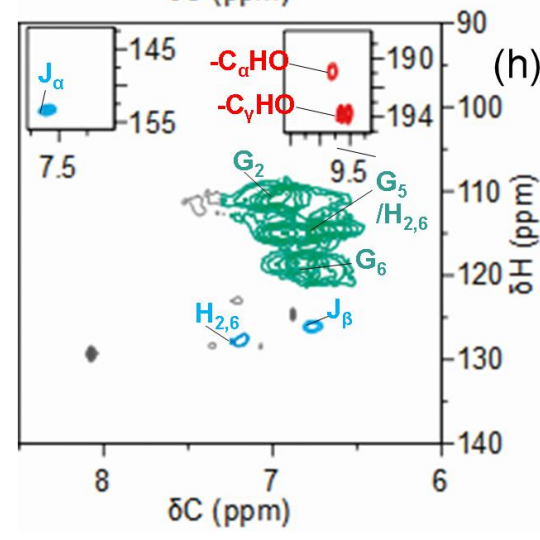

(j)

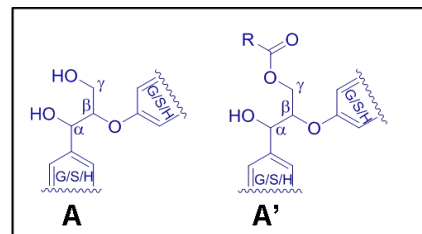

A

$A^{\prime}$

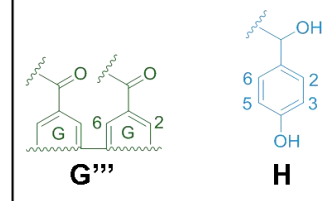

(b)
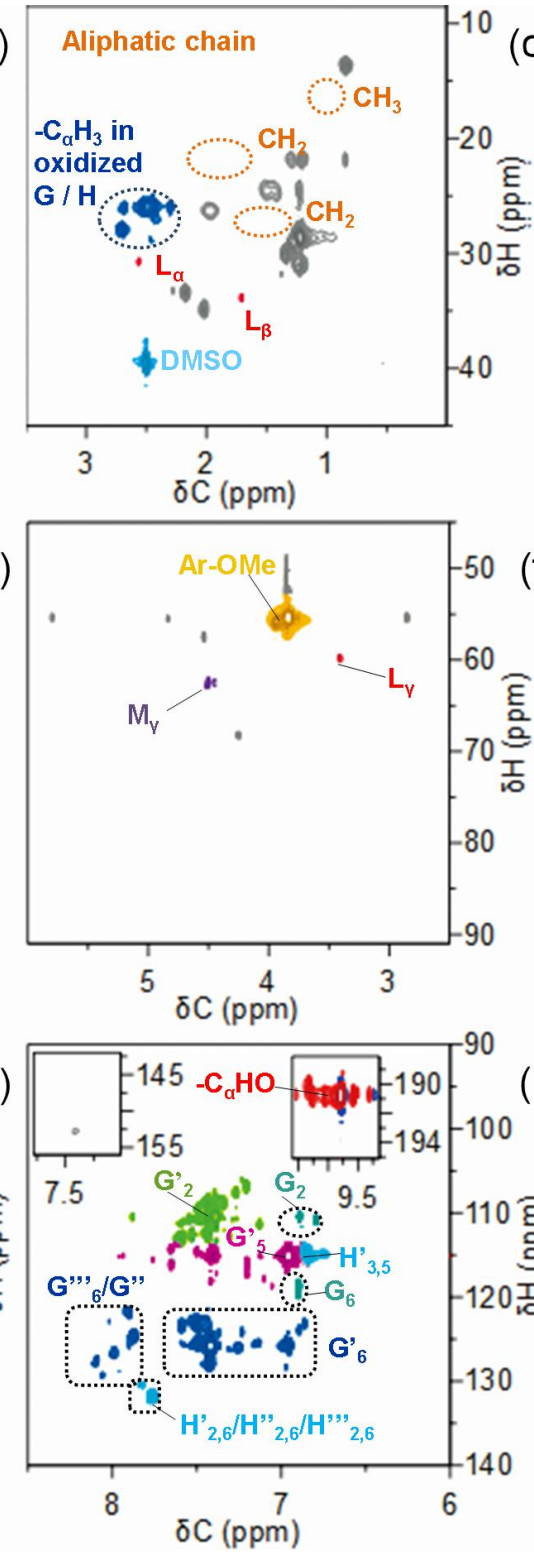

(c)

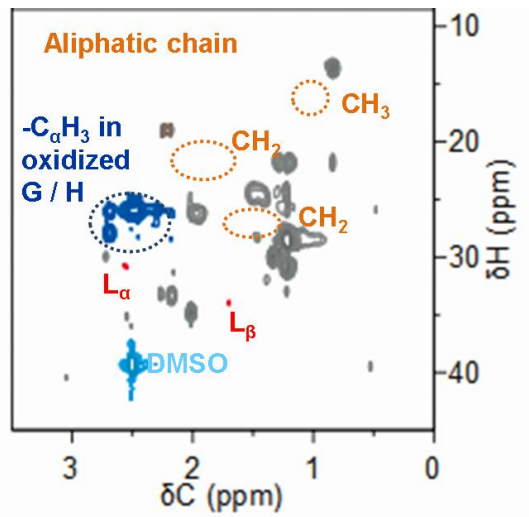

(f)

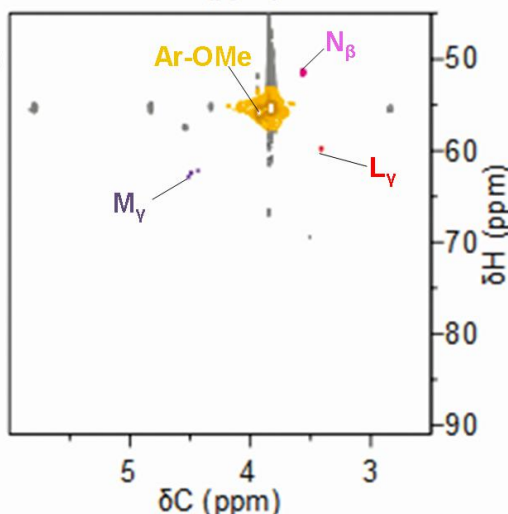

(i)

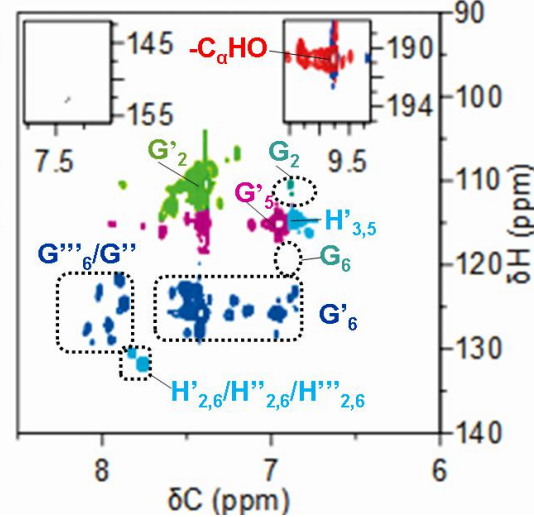

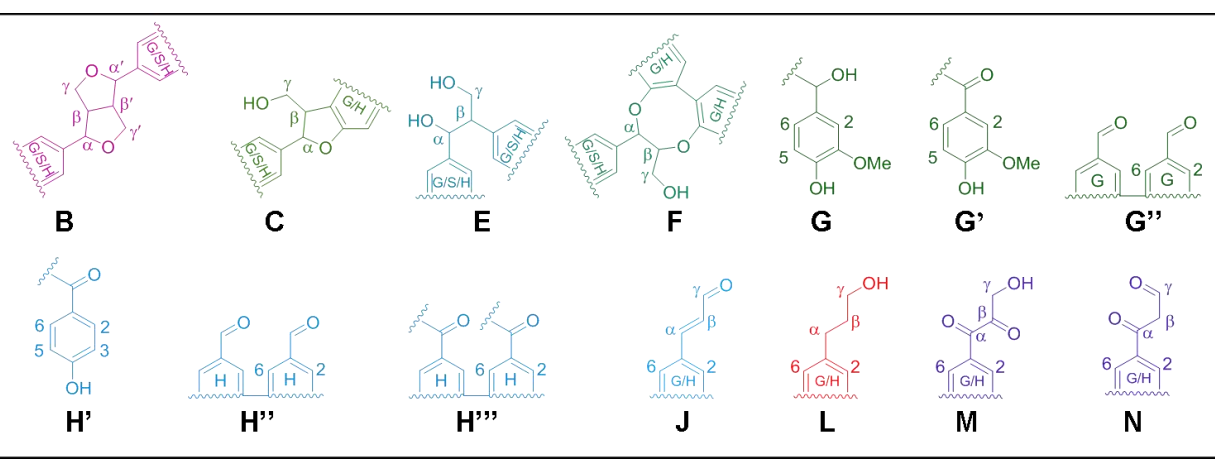

Figure 6. HSQC spectra of (a, d, g) pine MWL and OCF products in the chloroform phase that were prepared (b, e, h) in the absence, and (c, f, i) presence of $\mathrm{CuO}$ NPs under $\mathrm{O}_{2}$; and (j) main structure present in the MWLs and chloroform extracted lignin fragments. The assignments of labels can be found in Table S8. 


\subsection{Role of CuO NPs in protecting cellulose}

Use of $\mathrm{CuO}$ NPs during pine wood fractionation in alkaline not only improved fractionation and depolymerisation of lignin towards aromatic monomers, but it also to our surprise increased the retention of cellulose (in SR), irrespective of the atmosphere, viz. under $\mathrm{O}_{2}$ or $\mathrm{N}_{2}$. (Figure 5a-c, step 1; Figures 2 and 4). Moreover, the cellulose in SR showed higher CI and a smoother cellulose crystal surfaces (Figures S7e $\sim$ S7i, S8, S17 S20).

After pine wood fractionation in the presence of $\mathrm{CuO}$ NPs, SR was separated using a filter, while the spent catalyst ended in the filtrate due to its nano-sized dimensions. Prior to $\mathrm{CuO}$ NPs reuse (see later), we analyzed the presence and occurrence of $\mathrm{Cu}$ in the SR, which was first subjected to an ultrasonic treatment and a thoroughly wash with water to remove alkaline and spent catalyst. The green-blue color of the remaining cellulosic solid (Figure 7c) indicates presence of strongly adsorbed $\mathrm{Cu}(\mathrm{II})$ species. Deeper inspection of the green-blue color with diffuse reflectance spectroscopy in the UV to NIR spectrum showed the typical $d-d$ transition ${ }^{[79]}$ and a six coordination geometry typical of $\mathrm{Cu}$ (II) salts (Figure S48). XPS of the raw SR showed binding energies at 962.1, 955.2 and $934.8 \mathrm{eV}$, and two characteristic shake-up satellites at 944.1 and $941.1 \mathrm{eV}$, which are indicative of the presence of $\mathrm{Cu}$ (II) (Figure 8b). ${ }^{[80-82]}$ Suggestion of such $\mathrm{Cu}$ (II) ions might contradict the favorable $\mathrm{CuO}$ occurrence of $\mathrm{Cu}$, as predicted by Pourbaix, but the formation of acids and their strong complexation chemistry with $\mathrm{Cu}$ (II) can be invoked to explain its presence. In the solid, $\mathrm{Cu}$ (II) is most likely complexed with carboxylate groups at the surface of the cellulose matrix, and uniformly distributed as evidenced by the elemental mapping (Figure 8f). We and Lazic et al. have demonstrated earlier that cellulose obtained at soda cooking or under (metal free) OCF indeed has a carboxylate-functionalized surface. ${ }^{[83-85]}$ Note that acidification of SR with $\mathrm{HCl}$ turned the color into white leaving a blue-greenish colored filtrate.

To confirm the $\mathrm{Cu}$-cellulosic acid hypothesis, FT-IR was used (Figure 8a, Table S11). The SR before acidification showed a clearly carbonyl peak of 1644 $\mathrm{cm}^{-1}$ that is attributed to the carboxylate group, and which shifts to $1737 \mathrm{~cm}^{-1[86]}$, assigned to the carbonyl of the carboxylic acid group, upon acidification, depolymerisation indeed prevented by surface decoration of $\mathrm{Cu}$ ions through the formed surface carboxylate groups on cellulose (Scheme 3). In addition, also some $\mathrm{Cu}(\mathrm{I})$ was found in the unacidified $\mathrm{SR}$, as evidenced by the peaks of $\mathrm{Cu}$ (I) $(953.1 \mathrm{eV}$, $932.9 \mathrm{eV}$ ) on $\mathrm{Cu} 2 \mathrm{p}$ XPS spectrum and lattice spacing of $0.21 \mathrm{~nm}$ of $\mathrm{Cu}_{2} \mathrm{O}$ in HRTEM (Figure 8c and e), implying some reduction of $\mathrm{Cu}(\mathrm{II})$ into $\mathrm{Cu}(\mathrm{I})$ during the catalytic oxidation of lignocellulose. This supports the redox chemistry of the CuO NPs in the proposed catalytic oxidation mechanism (Scheme 2c). It is surprising to note here that the stabilization effect of $\mathrm{Cu}$ is less pronounced for $\mathrm{Cu}$ salts in OCF in comparison to the usage of $\mathrm{CuO} N P s$, despite the fact that the interaction of soluble $\mathrm{Cu}$ is at the origin of cellulose stabilization. We theorize that $\mathrm{CuO}$ NPs are required to oxidize the surface of cellulose, a functionalization that may be more difficult, if not possible, with $\mathrm{Cu}$ salts. (a)

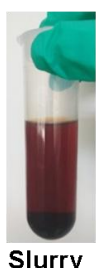

(b)

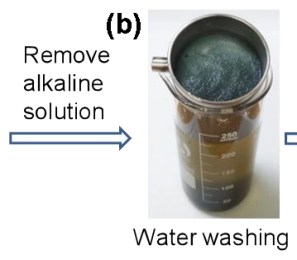

(c)

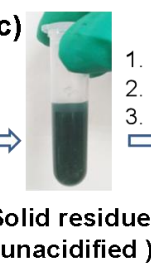

(d)

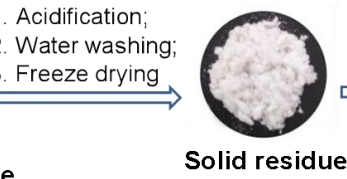

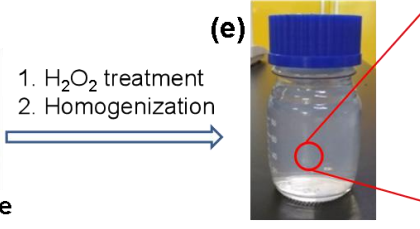

Nanocellulose

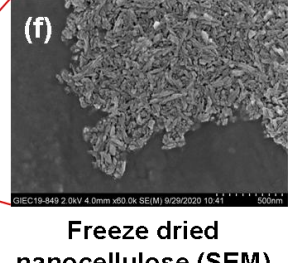

Figure 7. Separation process of SR and preparation procedure of cellulose nanocrystal. The slurry was obtained under following reaction conditions: $0.5 \mathrm{~g}$ pine, $7.5 \mathrm{wt} \% \mathrm{NaOH}, 0.1 \mathrm{~g} 40 \mathrm{~nm} \mathrm{CuO} \mathrm{NPs}, 160{ }^{\circ} \mathrm{C}, 60 \mathrm{~min}, 1 \mathrm{MPa} \mathrm{O}_{2}$, $400 \mathrm{rpm}$. 
(a)

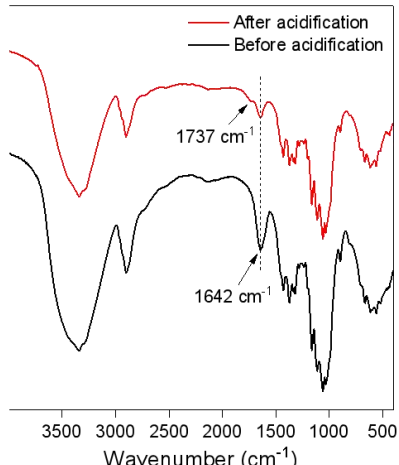

(e)

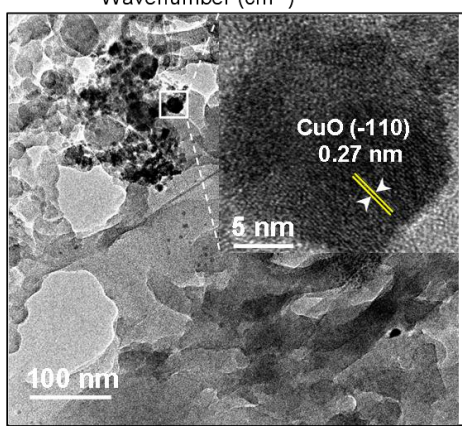

(b)
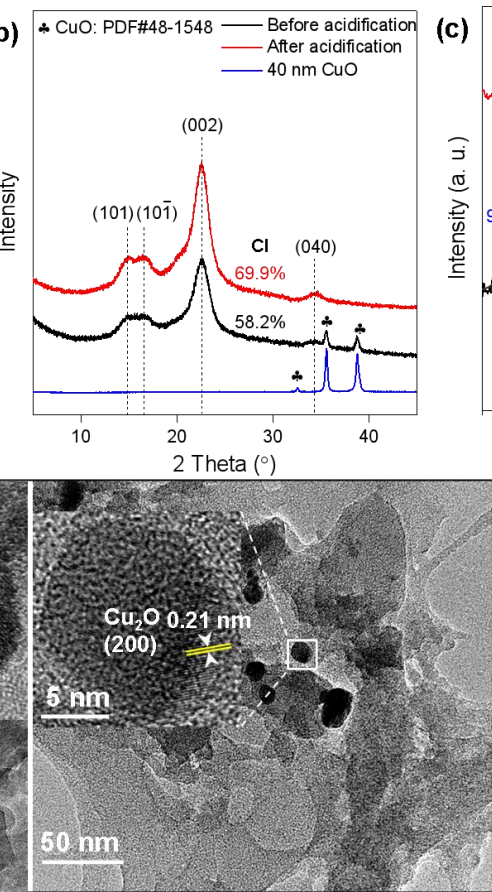

(c)

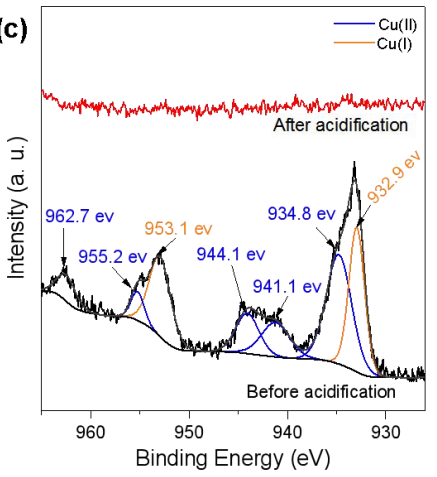

(f)

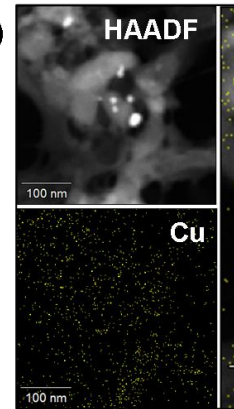

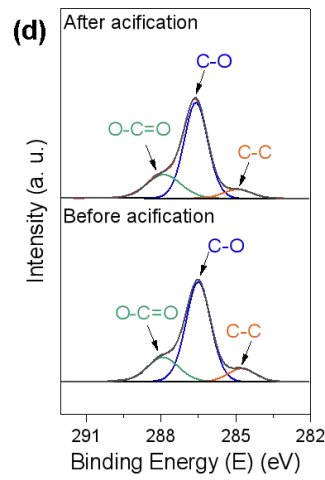

Binding Energy $(E)(e V)$

Cu / HAADF

Figure 8. (a) FTIR spectra, (b) XRD pattern, (c) Cu 2p XPS spectra, (d) C 1s XPS spectra of SR before and after acidification; (e) TEM images and (f) EDX mapping of SR before acidification.

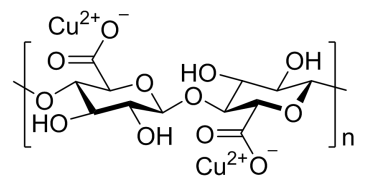

Scheme 3. Possible chemical structure of cellulose before acidification.

In addition, although the SR before acidified showed similar surface morphology to that after the acidification step (Figure S22), its CI significantly increased from $58.1 \%$ to $69.7 \%$ after acidification (Figure 8c), which attribute to the removal of the $\mathrm{Cu}(\mathrm{II})$ cations.

\subsection{Transformation of cellulose to cellulose nanocrystal}

The SR after reaction can be collected by sieving and acidification, as depicted in Figure 7a-d. The cellulose crystalline form was cellulose I (X-Ray analysis in Figure 8c, and FT-IR spectra in Figure 8a) and CI was high $(69.7 \%)$.

'Native' cellulose is used as a raw material to produce high-value nanocellulose, which has a wide range of applications in medicine delivery, ${ }^{[87]}$ catalyst support, ${ }^{[88]}$ and electronics devices ${ }^{[89]}$ [90-93] among others. We attempted the synthesize of nanocellulose by treating our cellulose product with $\mathrm{H}_{2} \mathrm{O}_{2}$, followed by homogenizing in a microfluidizer (seeMethods). Nice nanocrystals with a mean diameter of $56 \mathrm{~nm}$ (Figure S33), were thus obtained, demonstrating that the cellulose obtained from OCF has a broad application range that goes beyond production of chemicals, e.g., bioethanol, monosaccharide, ${ }^{[56,}$ 94, 95] alditol, ${ }^{[44]}$ and levulinic acid. ${ }^{[36]}$

\subsection{Stability and reusability of $\mathrm{CuO}$ NPs}

To assess the stability and reusability of $\mathrm{CuO}$ NPs, spent catalyst after simply washing step was tested in the next OCF of pine (see Methods).

Surprisingly, $\mathrm{CuO}$ NPs maintained their high activity after 4 recycles. Similar monophenolic yields (Figure S27a), Mw, PD and molecular weight distribution were obtained (Figure S5; Table 2) for all four reactions. Hemicellulose conversion and small acids yields, and product distribution of small acids, were also similar (Figure S27b). Moreover, there was also no obvious difference in the cellulose yield, its purity, 
crystal type and morphology (smooth surfaces) and CI (Figures 9, S27c, S23). After 4 runs, the spent catalyst remained its original $\mathrm{CuO}$ structure, as indicated by XRD pattern (Figure S9b). Despite observing very similar fractionation and depolymersiation results, only $70 \%$ of $\mathrm{CuO}$ NPs was ultimately collected due to losses of $\mathrm{Cu}$ in the complexation with the cellulose. This $\mathrm{Cu}$ (II) cations however can be readily collected by acidification of $\mathrm{SR}$ in the form of $\mathrm{CuCl}_{2}$ and $\mathrm{CuCl}$, which can be converted into $\mathrm{CuO}^{[82,96,97]}$ (Figure S32).

Table 2. Average molecular weights of chloroform extracted lignin fragments in the $\mathrm{CuO}$ NPs recycle experiments. $^{\text {a }}$

\begin{tabular}{|c|c|c|c|}
\hline CuO NPs use times & $M \mathrm{n}$ & $M \mathrm{w}$ & $P D$ \\
\hline 1 & 193 & 277 & 1.44 \\
\hline 2 & 198 & 255 & 1.29 \\
\hline 3 & 199 & 262 & 1.32 \\
\hline 4 & 194 & 264 & 1.36 \\
\hline
\end{tabular}

aReaction conditions: $0.5 \mathrm{~g}$ pine, $7.5 \mathrm{wt} \% \mathrm{NaOH}, 0.1$ g $40 \mathrm{~nm} \mathrm{CuO} \mathrm{NPs} \mathrm{(spent} \mathrm{catalyst} \mathrm{was} \mathrm{washed} \mathrm{to} \mathrm{pH} 7$ and used for the next recycle), $160^{\circ} \mathrm{C}, 60 \mathrm{~min}, 1 \mathrm{MPa}$ $\mathrm{O}_{2}, 400 \mathrm{rpm} . P D=M \mathrm{w} / M \mathrm{n}$.

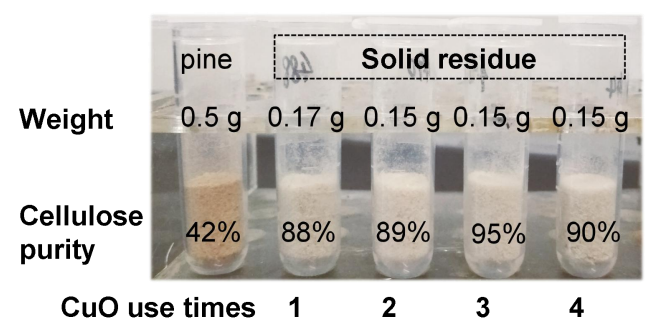

Figure 9. Picture of SR of pine of $\mathrm{CuO}$ NPs recycle experiments. Reaction conditions are similar to Table 2.

\subsection{Feedstock versatility using CuO NPs OCF for $r$ aromatic monomers and cellulose production}

OCF of a variety of biomass over CuO NPs was tested to demonstrate its versatility. Almost complete delignification $(\geqslant 95 \%)$ was achieved for all tested biomass feedstock (Table 3), resulting on a solid residue rich in cellulose crystals with smooth and clean surfaces(Figure S24). The lignin is fragmented into low Mws phenolics (Figure S6; Table S5), achieving high aromatic monomers yields (Figure S28a), especially in the cases of raw woody biomass.
For instance, the monophenolics yield of pine and eucalyptus were $33.8 \mathrm{wt} \%$ and $45.6 \mathrm{wt} . \%$, respectively, which were close to their theoretical yield (based on $\beta$ O-4) (Table 3). Yields were lower for raw herbs/grass biomass such as Chinese pennisetum, corn stalk, and bagasse, showing monophenolics yields of $21 \mathrm{wt} . \%, 23$ wt. $\%$ and 31 wt. $\%$, respectively.

According to HSQC analysis of MWL (Figures 7, S29 and S30; Table S9), the percentages of active $\beta-\mathrm{O}-4$ ether bond in all interlinkages of lignin in herb/grass biomass (Chinese pennisetum, bagasse and cornstalk) are slightly higher than that in soft (pine) or hard wood (eucalyptus). Correspondingly, the yield of phenolic monomers after OCF of Chinese pennisetum (53\%), bagasse $(56 \%)$ and cornstalk $(62 \%)$ are expected higher than that of pine (30\%) and eucalyptus (50\%) (theoretical values between brackets), which is not the case. Quite a few $\beta-O-4$ substructures in lignin of three herb/grass biomass are acetylated (A'), that may impact the oxidation chemistry under the applied conditions. Moreover, these herb/grass possess large amount of p-hydroxycinnamates such as p-coumarate (pCA) (16 39.4 per 100 Aromatic unit (AU)) and ferulate (FA) (2.3 4.9 per $100 \mathrm{AU})$ and lignan tricin (T) (1 3.6 per $100 \mathrm{AU})$, these units hard to be converted to aromatic aldehydes/ketone under the applied conditions (Figure S31b). Therefore, the monophenolics yields of herb/grass type of biomass are lower than that of woody biomass under our oxidative conditions. In addition, feedstock obtained from chemical pretreatment (residue of furfural production from corn stalk) gave low aromatic monomers yields (Table 3), which was attributed to the more condense (i.e. C-C bonds) structure of the lignin remaining after pretreatment.

Nitrobenzene oxidation, a chemical method for the evaluation of theoretical monophenolics yield of lignin, was used and compared with the results of OCF (Table 3). Surprisingly, all yields of monophenolics in the OCF with $\mathrm{CuO}$ NPs are higher than those using nitrobenzene method, indicating superior performance of OCF with $\mathrm{CuO}$ NPs. 
Typically, the aromatic aldehydes and their derived ketones accounted for a large portion of the monophenolics' composition (Figure S28a, Table 3). As indicated in Figure 10, the percentage of lignin units $(\mathrm{G}, \mathrm{S}, \mathrm{H})$ has a linear relationship with the percentage of monophenolics with corresponding lignin units, and the correlation coefficient is about 0.8 . This essentially means that the methoxy grops substituted at the arenes are less, if not all, effected by the radical oxidation chemistry. Therefore, the types and percentages of aldehydes and ketones are highly depended on original structure of lignin (Figures 7, S28 and S29; Tables S9 and S10).

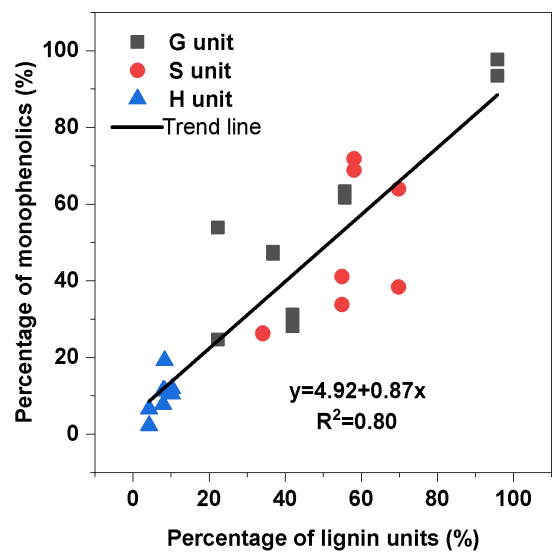

Figure 10. The relationship between percentages of lignin units with the percentages of monophenolics with corresponding lignin units. The percentages of lignin units were listed in Table S9. Percentages of monophenolics (G-type) $=\mathrm{N}_{(\text {vanillin }}+$ acetovanillone) $/$

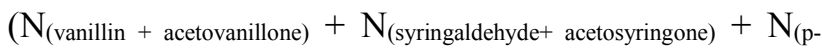
Hydroxy benzaldehyde+ $\mathrm{p}$-Hydroxyacetophenone)), where $\mathrm{N}$ refers to carbon molars of monophenolics (see data of Table 3). Percentages of $\mathrm{H}$ - or S-type monophenolics were calculated using the formula similar to that of G-type.

1 G $(31 \sim 77 \%)$ was the main product of all investigated biomass except for eucalyptus and cornstalk. 1S can be obtained with all lignocellulose biomass except for pine, and it is the main monophenolic product of eucalyptus (64\%) and cornstalk (52\%). In addition, 2G (5 25\%) and p-hydroxy benzaldehyde (1H) (4 19\%) were also products of pine and herbs/grass biomass (Chinese pennisetum, bagasse, corn stalk and its furfural residue) OCF . Moreover, all lignocellulosic biomass, except for pine, yielded small amount of acetosyringone (2S) (1 5\%), while all lignocellulosic biomass except for eucalyptus gave small amount of $\mathrm{p}$ hydroxyacetophenone $\quad(\mathbf{2 H}) \quad(2 \sim 4 \%) . \quad$ Other monophenolics like guaiacol, phenol were also analyzed but their yields were too low to be accurately quantified.

In addition to raw lignocellulosic biomass, technical lignin (here: alkalin lignin of pine) was used as substrate of OCF. The results shows very little formation of monomers, confirming the importance of ether functionality in the OCF as starting points of the depolymerisation towards monomers. Therefore, OCF on native lignin delivers, besides a clean and crystalline cellulose, much higher yield of monomeric phenolics, to the best of our knowledge, being highest in presence of $\mathrm{CuO}$ NPs.

The cellulose yields of pine, eucalyptus, Chinese pennisetum, cornstalk and bagasse were $69.7 \%, 61.1 \%$ $79.4 \%$, 55.2\%, 67.4\%, respectively, which increased by $50 \%, 191 \%, 166 \%, 86 \%, 151 \%, 435 \%$, respectively, due to the usage of CuO NPs (Figure 11b). Moreover, the SRs of all lignocllulosic biomass with $\mathrm{CuO}$ NPs showed all the same cellulose crystal form and similar or higher CI than that obtained in absence of catalyst (Figure S10). In addition to better yield, the crystals have nice smooth and intact surfaces, as opposed to the cellulose remaining obtained in absence of $\mathrm{CuO}$ NPs. (Figure S24).

The retention of more cellulose obviously led to lower yields of the small acids as apparent from the data in Figure 11a. Nevertheless, more oxalic acid was obtained, suggesting the oxidation chemistry in presence of $\mathrm{CuO}$ NPs have a preference for its formation. Interestingly, conversion of technical lignin, that is without presence of cellulose, also forms large contents of small acids, albeit different ones (no gluconic acid, or lactic acid, or succinic acid was formed), which is due to deep oxidation of the aromatics and its end groups. That means that solubilization of sugars, and its preferred oxidation, somehow protects the lignin fragments from deeper 
Table 3. OCF of various biomass into monophenolics over $\mathrm{CuO}$ NPs.
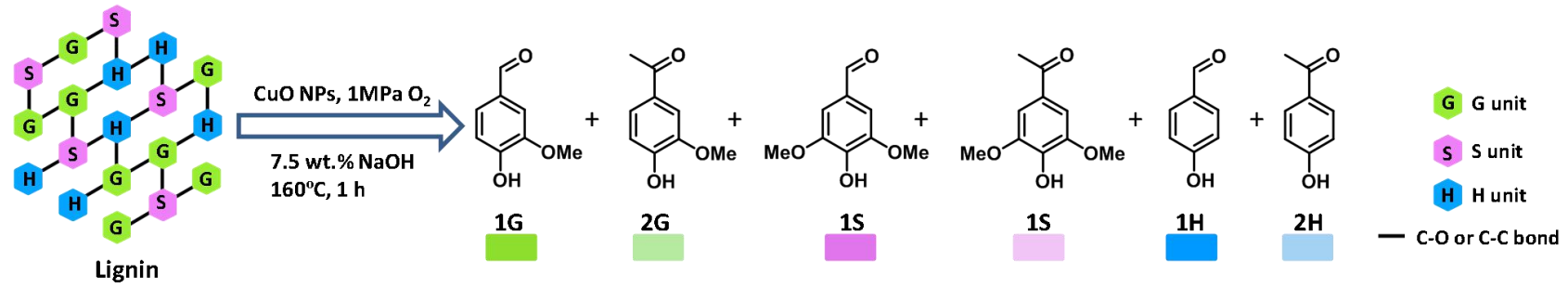

\begin{tabular}{|c|c|c|c|c|c|c|c|}
\hline Raw material & Pine & Eucalyptus & $\begin{array}{c}\text { Chinese } \\
\text { pennisetum }\end{array}$ & Corn stalk & Bagasse & $\begin{array}{l}\text { Furfural } \\
\text { residue }\end{array}$ & $\begin{array}{c}\text { Alkaline } \\
\text { lignin }\end{array}$ \\
\hline Delignification $(\%)$ & 95 & 100 & 100 & 100 & 100 & 100 & - \\
\hline $\begin{array}{l}\text { Monophenolics yield } \\
(\mathrm{wt} \%)\end{array}$ & 33.8 & $45.6^{\mathrm{b}}$ & 25.1 & $22.6^{\mathrm{b}}$ & 30.6 & 9.5 & 9 \\
\hline $\begin{array}{c}\text { Theoretical } \\
\text { monophenolics yield } \\
\qquad \%)^{c}\end{array}$ & 30 & 50 & 53 & 56 & 62 & - & - \\
\hline $\begin{array}{c}\text { Nitrobenzene } \\
\text { oxidation }(w t \%)^{d}\end{array}$ & 25.6 & 39.3 & 17 & 22.1 & 28.8 & 8 & 7 \\
\hline $\begin{array}{l}\text { Monophenolics } \\
\text { distribution }\end{array}$ & $6 \%$ & $8 \% \quad 23 \%$ & $25 \%$ & $\begin{array}{r}12 \%{ }^{2 \%}{ }^{20} \\
\\
52 \%\end{array}$ & $\begin{array}{l}17 \% \\
5 \%\end{array}$ & 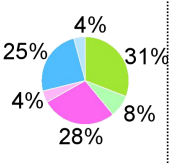 & $19 \%$ \\
\hline
\end{tabular}

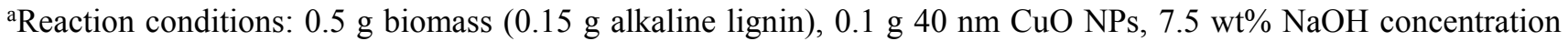
$(25 \mathrm{~mL}), 160{ }^{\circ} \mathrm{C}, 60 \mathrm{~min}, 400 \mathrm{rpm}, 1 \mathrm{MPa} \mathrm{O}{ }_{2} .{ }^{\mathrm{b}} 40 \mathrm{~nm} \mathrm{CuO}$ NPs loading: $0.2 \mathrm{~g}$. ${ }^{\mathrm{c}}$ Theoretical monophenol yields were calculated according to previous method ${ }^{[98]}$ using the data (ether bond abundance in lignin) of Table S9. ${ }^{\mathrm{d}}$ Reaction condition: $0.5 \mathrm{~g}$ biomass (0.15 g alkaline lignin), $3 \mathrm{~mL}$ nitrobenzene, $27 \mathrm{~mL} \mathrm{NaOH}$ aqueous solution $(2 \mathrm{~N}), 170^{\circ} \mathrm{C}, 2 \mathrm{~h}, 400 \mathrm{rpm}$.
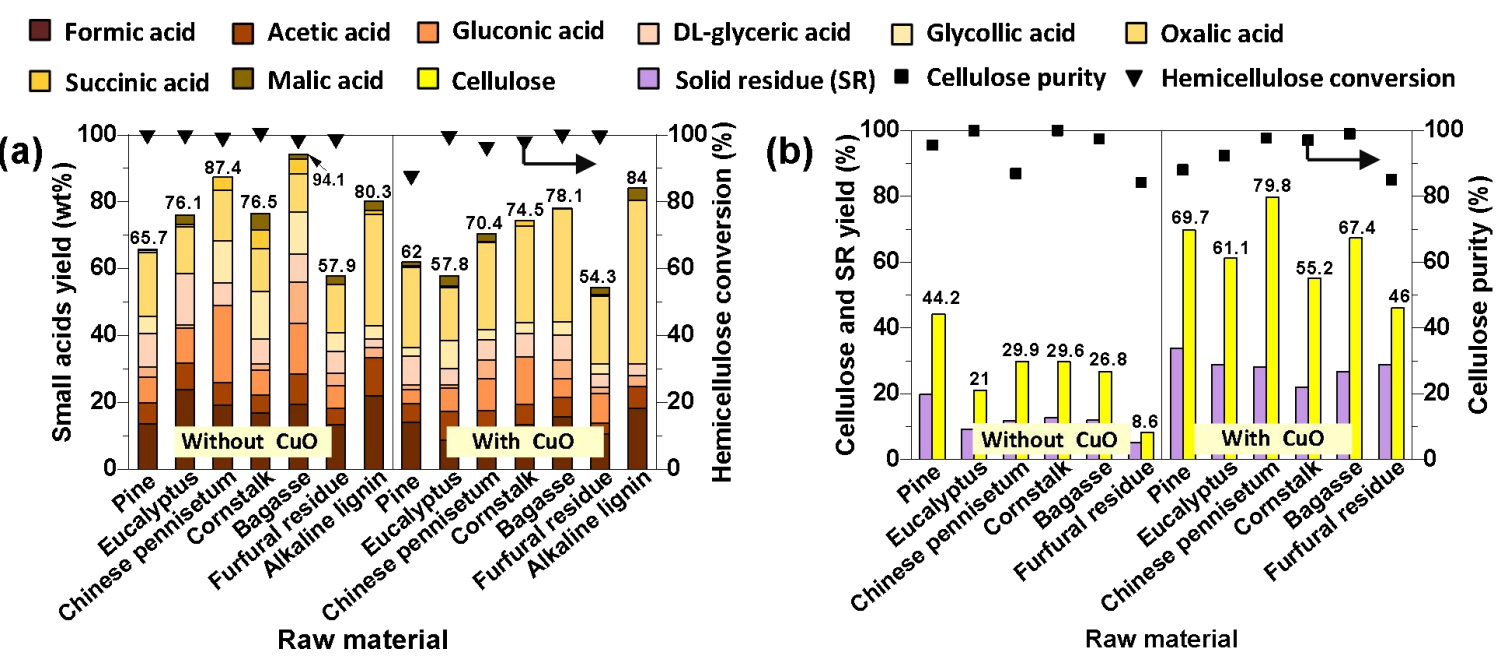

Figure 11. Yield of small acids, cellulose and SR by OCF of various biomass over CuO NPs. Reaction conditions:

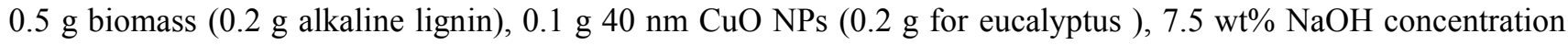
$(25 \mathrm{~mL}), 160^{\circ} \mathrm{C}, 60 \mathrm{~min}, 400 \mathrm{rpm}, 1 \mathrm{MPa} \mathrm{O}_{2}$.

oxidation, another good reason to apply lignin-first strategies such as OCF.
In order to evaluate the valorization of biomass by OCF in the presence of $\mathrm{CuO} \mathrm{NPs,} \mathrm{the} \mathrm{weight} \mathrm{yield} \mathrm{and}$ 
carbon efficiencies of the different biomasses are summarized (see Methods for the calculation). As can be seen in Figure 12, total weight yield of products with $\mathrm{CuO}$ NPs are higher or equal to those without $\mathrm{CuO}$ NPs, wherein the yield of cellulose and monophenolics of lignocellulose biomass increased at the expense of the small acids yield. Notably, the carbon efficiencies of OCF of raw biomass like pine, eucalyptus and furfural residue are the highest reported today, as long as $\mathrm{CuO}$ NPs is used in their OCF.. For instance a comparison with the fractionation results in absence of $\mathrm{CuO}$ NPs shows a carbon efficiency of eucalyptus increase from $68 \mathrm{C} \%$ to $78 \mathrm{C} \%$, and a monophenol yield and cellulose yield increase from 8 $\mathrm{C} \%$ to $10.9 \mathrm{C} \%$ and $8.5 \mathrm{C} \%$ to $24.6 \mathrm{C} \%$, respectively; the small acids yield decreased from $42.3 \mathrm{C} \%$ to 38.4 $\mathrm{C} \%$.

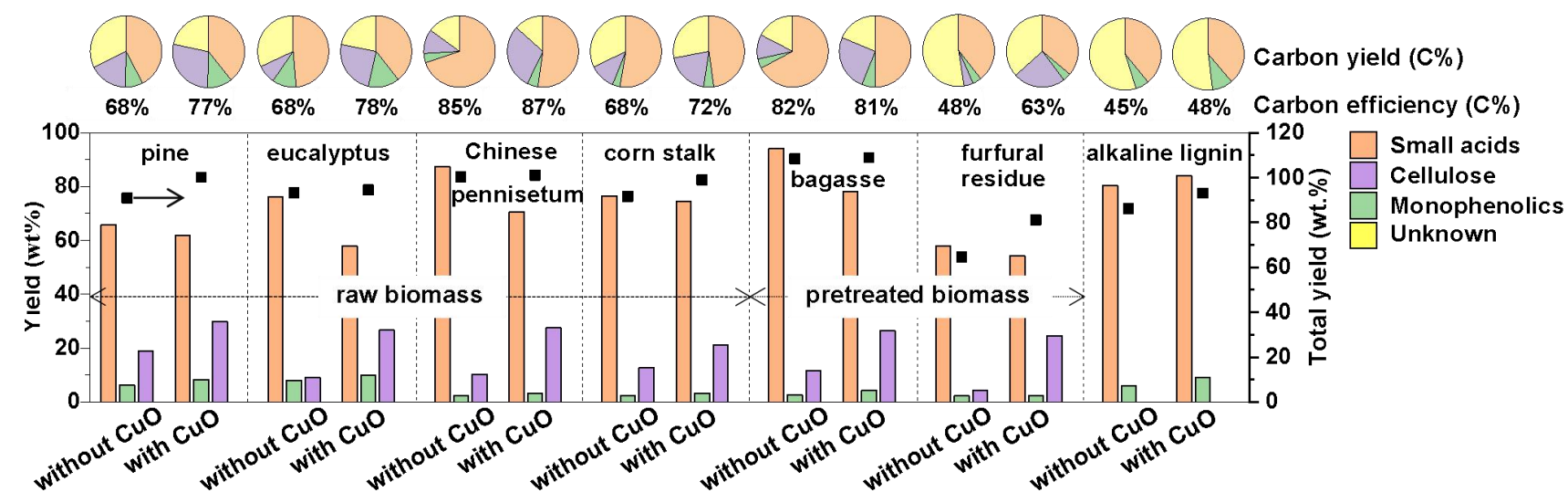

Figure 12. Weight yield and carbon yield of products and carbon efficiency during OCF of different biomass with and without $\mathrm{CuO}$ NPs. Reaction condition: $0.5 \mathrm{~g}$ biomass (technical lignin, $0.15 \mathrm{~g}$ ), $7.5 \mathrm{wt} . \% \mathrm{NaOH}(25 \mathrm{~mL}), 160^{\circ}$ C, $60 \mathrm{~min}, 1 \mathrm{MPa} \mathrm{O}$, $400 \mathrm{rpm}$; CuO NPs loading : $0.1 \mathrm{~g}$ (0.2 g for eucalyptus and cornstalk).

\section{Conclusions}

In summary, we have developed an OCF strategy in presence of $\mathrm{CuO}$ NPs for the co-production of highvalue chemicals (aromatic aldehydes and ketones) and material (pure cellulose) from lignocellulose. $\mathrm{CuO}$ NPs exhibit excellent performance in the selective depolymerisation through interlinkage cleavage and oxidation of lignin (fragments), giving high yields of monomeric phenolics such as vanillin and syringaldehyde, with a maximum preservation of a white, high quality cellulose, that is useful for high value nanocellulose production. The latter is likely due to surface oxidation and protection by complexation with $\mathrm{Cu}$ cations. The OCF method with $\mathrm{CuO}$ is a versatile method, able to fractionate and depolymerize a different biomass feedstock, the phenolics product composition being in line with the original lignin structure of the biomass source. The total carbon efficiency of the biomass conversion to useful products using the $\mathrm{CuO}$ NPs OCF process is very high and close to $90 \mathrm{C} \%$, suggesting that the OCF strategy is a promising technology towards full biomass valorization.

\section{Methods}

See Materials and methods in the supporting information.

\section{Data availability}

The data supporting the findings of this study are available from the corresponding authors upon reasonable request.

\section{Author contributions}

Y.Z. and C.W. conceived the project and designed the experiments. Y.Z. and L.L. performed the experiments and analysed the data with the input from Y.L., C.W and B. S. Y.Z. wrote the manuscript with input from 
all other authors. C.W. and B.S. supervised the project.

\section{Competing interests}

The authors declare no competing interests.

\section{Acknowledgments}

The authors acknowledge the financial support from the National Key R\&D Program of China (2018YFB1501504), Key R\&D Program of Guangdong Province (No. 2020B1111570001) and and National Natural Science Foundation of China (No. 51776206).

\section{Reference}

1. C. Z. Li, X. C. Zhao, A. Q. Wang, G. W. Huber and T. Zhang, Chemical Reviews, Catalytic Transformation of Lignin for the Production of Chemicals and Fuels, 2015, 115, 11559-11624.

2. J. Zakzeski, P. C. A. Bruijnincx, A. L. Jongerius and B. M. Weckhuysen, Chemical Reviews, The Catalytic Valorization of Lignin for the Production of Renewable Chemicals, 2010, 110, 3552-3599.

3. W. Schutyser, T. Renders, S. Van den Bosch, S.F. Koelewijn, G. T. Beckham and B. F. Sels, Chemical Society Reviews, Chemicals from lignin: an interplay of lignocellulose fractionation, depolymerisation, and upgrading, 2018, 47, 852-908.

4. C. P. Xu, R. A. D. Arancon, J. Labidi and R. Luque, Chemical Society Reviews, Lignin depolymerisation strategies: towards valuable chemicals and fuels, 2014, 43, 7485-7500.

5. R. Behling, S. Valange and G. Chatel, Green Chemistry, Heterogeneous catalytic oxidation for lignin valorization into valuable chemicals: What results? What limitations? What trends?, 2016, 18, 1839-1854.

6. Y. H. Liao, Q. Y. Liu, T. J. Wang, J. X. Long, L. L. Ma and Q. Zhang, Green Chemistry, Zirconium phosphate combined with $\mathrm{Ru} / \mathrm{C}$ as a highly efficient catalyst for the direct transformation of cellulose to C6 alditols, 2014, 16, 3305-3312.

7. N. Shi, Q. Y. Liu, Q. Zhang, T. J. Wang and L. L. Ma, Green Chemistry, High yield production of 5-hydroxymethylfurfural from cellulose by high concentration of sulfates in biphasic system, 2013, 15, 1967-1974.

8. X. H. Liu, X. D. Liu, G. Y. Xu, Y. Zhang, C. G. Wang, Q. Lu and L. L. Ma, Green Chemistry, Highly efficient catalytic conversion of cellulose into acetol over Ni-Sn supported on nanosilica and the mechanism study, 2019, 21, 56475656.

9. Q. Y. Liu, H. Y. Wang, H. S. Xin, C. G. Wang, L. Yan, Y. X. Wang, Q. Zhang, X. H. Zhang, Y. $\mathrm{Xu}, \mathrm{G} . \mathrm{W}$. Huber and L. L. Ma, Chemsuschem, Selective Cellulose Hydrogenolysis to Ethanol Using Ni@C Combined with Phosphoric Acid Catalysts, 2019, 12, 3977-3987.

10. Y. H. Liao, B. O. de Beeck, K. Thielemans, T. Ennaert, J. Snelders, M. Dusselier, C. M. Courtin and B. F. Sels, Molecular Catalysis, The role of pretreatment in the catalytic valorization of cellulose, 2020, 487 .

11. A. Q. Wang and T. Zhang, Accounts of Chemical Research, One-Pot Conversion of Cellulose to Ethylene Glycol with Multifunctional Tungsten-Based Catalysts, 2013, 46, 1377-1386.

12. Y. B. Huang and Y. Fu, Green Chemistry, Hydrolysis of cellulose to glucose by solid acid catalysts, 2013, 15, 1095-1111.

13. Y. Xiong, Z. H. Zhang, X. Wang, B. Liu and J. T. Lin, Chemical Engineering Journal, Hydrolysis of cellulose in ionic liquids catalyzed by a magnetically-recoverable solid acid catalyst, 2014, 235, 349-355.

14. T. Vangeel, W. Schutyser, T. Renders and B. F. Sels, Top Curr Chem (Cham), Perspective on Lignin Oxidation: Advances, Challenges, and Future Directions, 2018, 376, 30.

15. V. Menon and M. Rao, Progress in Energy and Combustion Science, Trends in bioconversion of lignocellulose: Biofuels, platform chemicals 
\& biorefinery concept, 2012, 38, 522-550.

16. Y. H. Liao, S. F. Koelewijn, G. Van den Bossche, J. Van Aelst, S. Van den Bosch, T. Renders, K. Navare, T. Nicolai, K. Van Aelst, M. Maesen, H. Matsushima, J. M. Thevelein, K. Van Acker, B. Lagrain, D. Verboekend and B. F. Sels, Science, A sustainable wood biorefinery for low-carbon footprint chemicals production, 2020, 367, 1385-+.

17. Z. Sun, G. Bottari, A. Afanasenko, M. C. A. Stuart, P. J. Deuss, B. Fridrich and K. Barta, Nature Catalysis, Complete lignocellulose conversion with integrated catalyst recycling yielding valuable aromatics and fuels, 2018, 1, 82-92.

18. C. W. Lahive, P. J. Deuss, C. S. Lancefield, Z. H. Sun, D. B. Cordes, C. M. Young, F. Tran, A. M. Slawin, J. G. de Vries, P. C. J. Kamer, N. J. Westwood and K. Barta, Journal of the American Chemical Society, Advanced Model Compounds for Understanding Acid-Catalyzed Lignin Depolymerization: Identification of Renewable Aromatics and a Lignin-Derived Solvent, 2016, 138, 8900-8911.

19. Z. Sun, J. Cheng, D. Wang, T. Q. Yuan, G. Song and K. Barta, ChemSusChem, Downstream Processing Strategies for Lignin-First Biorefinery, 2020, 13, 5199-5212.

20. Y. M. Questell-Santiago, M. V. Galkin, K. Barta and J. S. Luterbacher, Nature Reviews Chemistry, Stabilization strategies in biomass depolymerization using chemical functionalization, 2020, 4, 311-330.

21. M. M. Abu-Omar, K. Barta, G. T. Beckham, J. S. Luterbacher, J. Ralph, R. Rinaldi, Y. RománLeshkov, J. S. M. Samec, B. F. Sels and F. Wang, Energy \& Environmental Science, Guidelines for performing lignin-first biorefining, $\mathbf{2 0 2 0}$.

22. Z. Cao, M. Dierks, M. T. Clough, I. B. Daltro de Castro and R. Rinaldi, Joule, A Convergent Approach for a Deep Converting Lignin-First Biorefinery Rendering High-Energy-Density Drop-in Fuels, 2018, 2, 1118-1133.
23. R. Rinaldi, R. Jastrzebski, M. T. Clough, J. Ralph, M. Kennema, P. C. A. Bruijnincx and B. M. Weckhuysen, Angew. Chem. Int. Ed., Paving the Way for Lignin Valorisation: Recent Advances in Bioengineering, Biorefining and Catalysis, 2016, 55, 8164-8215.

24. D. Verboekend, Y. H. Liao, W. Schutyser and B. F. Sels, Green Chemistry, Alkylphenols to phenol and olefins by zeolite catalysis: a pathway to valorize raw and fossilized lignocellulose, 2016, 18, 297-306.

25. Y. H. Liao, M. d'Halluin, E. Makshina, D. Verboekend and B. F. Sels, Applied Catalysis BEnvironmental, Shape selectivity vapor-phase conversion of lignin-derived 4-ethylphenol to phenol and ethylene over acidic aluminosilicates: Impact of acid properties and pore constraint, 2018, 234, 117-129.

26. Y. H. Liao, R. Y. Zhong, E. Makshina, M. d'Halluin, Y. van Limbergen, D. Verboekend and B. F. Sels, Acs Catalysis, Propylphenol to Phenol and Propylene over Acidic Zeolites: Role of Shape Selectivity and Presence of Steam, 2018, 8, 7861-7878.

27. D. R. Vardon, M. A. Franden, C. W. Johnson, E. M. Karp, M. T. Guarnieri, J. G. Linger, M. J. Salm, T. J. Strathmann and G. T. Beckham, Energy \& Environmental Science, Adipic acid production from lignin, 2015, 8, 617-628.

28. R. S. Ma, Y. Xu and X. Zhang, Chemsuschem, Catalytic Oxidation of Biorefinery Lignin to Value-added Chemicals to Support Sustainable Biofuel Production, 2015, 8, 24-51.

29. V. Tarabanko and N. Tarabanko, International Journal of Molecular Sciences, Catalytic Oxidation of Lignins into the Aromatic Aldehydes: General Process Trends and Development Prospects, 2017, 18, 2421.

30. M. Fache, B. Boutevin and S. Caillol, ACS Sustainable Chem. Eng., Vanillin Production from Lignin and Its Use as a Renewable Chemical, 2016, 4, 35-46.

31. M. Fache, B. Boutevin and S. Caillol, European Polymer Journal, Vanillin, a key-intermediate of 
biobased polymers, 2015, 68, 488-502.

32.

W. Schutyser, J. S. Kruger, A. M. Robinson, R. Katahira, D. G. Brandner, N. S. Cleveland, A. Mittal, D. J. Peterson, R. Meilan, Y. RománLeshkov and G. T. Beckham, Revisiting alkaline aerobic lignin oxidation, 2018.

33. V. E. Tarabanko, K. L. Kaygorodov, E. A. Skiba, N. Tarabanko, Y. V. Chelbina, O. V. Baybakova, B. N. Kuznetsov and L. Djakovitch, Journal of Wood Chemistry and Technology, Processing Pine Wood into Vanillin and Glucose by Sequential Catalytic Oxidation and Enzymatic Hydrolysis, 2017, 37, 43-51.

34. A. G. Demesa, A. Laari, I. Turunen and M. Sillanpää, Chem. Eng. Technol. , Alkaline Partial Wet Oxidation of Lignin for the Production of Carboxylic Acids, 2015, 38, 2270-2278.

35. P. C. R. Pinto, C. E. Costa and A. E. Rodrigues, Industrial \& Engineering Chemistry Research, Oxidation of Lignin from Eucalyptus globulus Pulping Liquors to Produce Syringaldehyde and Vanillin, 2013, 52, 4421-4428.

36. Y. Zhu, Y. Liao, W. Lv, J. Liu, X. Song, L. Chen, C. Wang, B. F. Sels and L. Ma, ACS Sustainable Chemistry \& Engineering, Complementing Vanillin and Cellulose Production by Oxidation of Lignocellulose with Stirring Control, 2020, 8, 2361-2374.

37. C. Wang, M. Li and Y. Fang, Green Chemistry, Upgrading of pyrolytic lignin into hexamethylbenzene with high purity: demonstration of the "all-to-one" biochemical production strategy in thermo-chemical conversion, 2019, 21, 1000-1005.

38. F.-X. Collard and J. Blin, Renewable and Sustainable Energy Reviews, A review on pyrolysis of biomass constituents: Mechanisms and composition of the products obtained from the conversion of cellulose, hemicelluloses and lignin, 2014, 38, 594-608.

39. A. Narani, R. K. Chowdari, C. Cannilla, G. Bonura, F. Frusteri, H. J. Heeres and K. Barta, Green Chemistry, Efficient catalytic hydrotreatment of Kraft lignin to alkylphenolics using supported NiW and NiMo catalysts in supercritical methanol, 2015, 17, 5046-5057.

40. J. Ni, Y. T. Wu, F. Tao, Y. Peng and P. Xu, J Am Chem Soc, A Coenzyme-Free Biocatalyst for the Value-Added Utilization of Lignin-Derived Aromatics, 2018, 140, 16001-16005.

41. T. Kleine, J. Buendia and C. Bolm, Green Chem., Mechanochemical degradation of lignin and wood by solvent-free grinding in a reactive medium, 2013, 15, 160-166.

42. J. S. Luterbacher, A. Azarpira, A. H. Motagamwala, F. Lu, J. Ralph and J. A. Dumesic, Energy \& Environmental Science, Lignin monomer production integrated into the $\gamma$-valerolactone sugar platform, 2015, 8, 26572663.

43. R. Y. Shu, J. X. Long, Y. Xu, L. L. Ma, Q. Zhang, T. J. Wang, C. G. Wang, Z. Q. Yuan and Q. Y. $\mathrm{Wu}$, Bioresource Technology, Investigation on the structural effect of lignin during the hydrogenolysis process, 2016, 200, 14-22.

44. S. Van den Bosch, W. Schutyser, R. Vanholme, T. Driessen, S. F. Koelewijn, T. Renders, B. De Meester, W. J. J. Huijgen, W. Dehaen, C. M. Courtin, B. Lagrain, W. Boerjan and B. F. Sels, Energy \& Environmental Science, Reductive lignocellulose fractionation into soluble ligninderived phenolic monomers and dimers and processable carbohydrate pulps, 2015, 8, 17481763.

45. L. Shuai and B. Saha, Green Chemistry, Towards high-yield lignin monomer production, 2017, 19, 3752-3758.

46. B. Wang, X. J. Shen, J. L. Wen, L. Xiao and R. C. Sun, International Journal of Biological Macromolecules, Evaluation of organosolv pretreatment on the structural characteristics of lignin polymers and follow-up enzymatic hydrolysis of the substrates from Eucalyptus wood, 2017, 97, 447-459.

47. K. Ninomiya, K. Ochiai, M. Eguchi, K. Kuroda, Y. Tsuge, C. Ogino, T. Taima and K. Takahashi, Industrial Crops and Products, Oxidative 
depolymerization potential of biorefinery lignin obtained by ionic liquid pretreatment and subsequent enzymatic saccharification of eucalyptus, 2018, 111, 457-461.

48. K. H. Kim, B. A. Simmons and S. Singh, Green Chemistry, Catalytic transfer hydrogenolysis of ionic liquid processed biorefinery lignin to phenolic compounds, 2017, 19, 215-224.

49. C. Alvarez-Vasco, R. S. Ma, M. Quintero, M. Guo, S. Geleynse, K. K. Ramasamy, M. Wolcott and X. Zhang, Green Chemistry, Unique lowmolecular-weight lignin with high purity extracted from wood by deep eutectic solvents (DES): a source of lignin for valorization, $\mathbf{2 0 1 6}$, 18, 5133-5141.

50. C. Li, M. Zheng, A. Wang and T. Zhang, Energy Environ. Sci., One-pot catalytic hydrocracking of raw woody biomass into chemicals over supported carbide catalysts: simultaneous conversion of cellulose, hemicellulose and lignin, 2012, 5, 6383-6390.

51. T. Renders, S. Van den Bosch, S.-F. Koelewijn, W. Schutyser and B. F. Sels, Energy \& Environmental Science, Lignin-first biomass fractionation, the advent of active stabilisation strategies, 2017, 10, 1551-1557

52. X. Luo, Y. Li, N. K. Gupta, B. Sels, J. Ralph and L. Shuai, Angew Chem Int Ed Engl, Protection Strategies Enable Selective Conversion of Biomass, 2020, 59, 11704-11716.

53. Y. Liu, G. Luo, H. H. Ngo, W. Guo and S. Zhang, Bioresour Technol, Advances in thermostable laccase and its current application in lignin-first biorefinery: A review, 2020, 298, 122511.

54. K. Van Aelst, E. Van Sinay, T. Vangeel, E. Cooreman, G. Van den Bossche, T. Renders, J. Van Aelst, S. Van den Bosch and B. F. Sels, Chemical Science, Reductive catalytic fractionation of pine wood: elucidating and quantifying the molecular structures in the lignin oil, 2020, 11, 11498-11508.

55. S. Van den Bosch, T. Renders, S. Kennis, S. F. Koelewijn, G. Van den Bossche, T. Vangeel, A.
Deneyer, D. Depuydt, C. M. Courtin, J. M. Thevelein, W. Schutyser and B. F. Sels, Green Chemistry, Integrating lignin valorization and bio-ethanol production: on the role of $\mathrm{Ni}$ A12O3catalyst pellets during lignin-first fractionation, 2017, 19, 3313-3326.

56. T. Renders, E. Cooreman, S. Van den Bosch, W. Schutyser, S. F. Koelewijn, T. Vangeel, A. Deneyer, G. Van den Bossche, C. M. Courtin and B. F. Sels, Green Chemistry, Catalytic lignocellulose biorefining in n-butanol/water: a one-pot approach toward phenolics, polyols, and cellulose, 2018, 20, 4607-4619.

57. T. Parsell, S. Yohe, J. Degenstein, T. Jarrell, I. Klein, E. Gencer, B. Hewetson, M. Hurt, J. I. Kim, H. Choudhari, B. Saha, R. Meilan, N. Mosier, F. Ribeiro, W. N. Delgass, C. Chapple, H. I. Kenttämaa, R. Agrawal and M. M. AbuOmar, Green Chemistry, A synergistic biorefinery based on catalytic conversion of lignin prior to cellulose starting from lignocellulosic biomass, 2015, 17, 1492-1499.

58. I. Kumaniaev, E. Subbotina, J. Sävmarker, M. Larhed, M. V. Galkin and J. S. M. Samec, Green Chemistry, Lignin depolymerization to monophenolic compounds in a flow-through system, 2017, 19, 5767-5771.

59. X. Wu, X. Fan, S. Xie, J. Lin, J. Cheng, Q. Zhang, L. Chen and Y. Wang, Nature Catalysis, Solar energy-driven lignin-first approach to full utilization of lignocellulosic biomass under mild conditions, 2018, 1, 772-780.

60. L. Shuai, M. T. Amiri, Y. M. Questell-Santiago, F. Heroguel, Y. Li, H. Kim, R. Meilan, C. Chapple, J. Ralph and J. S. Luterbacher, Science, Formaldehyde stabilization facilitates lignin monomer production during biomass depolymerization, 2016, 354, 329-333.

61. W. Lan, M. T. Amiri, C. M. Hunston and J. S. Luterbacher, Angew. Chem. Int. Ed., Protection Group Effects During alpha,gamma-Diol Lignin Stabilization Promote High-Selectivity Monomer Production, 2017, 57, 1-6.

62. Y. Li, J. Chang and Y. Ouyang, Advanced 
Materials Research, Selective Production of Aromatic Aldehydes from Lignin by Metalloporphyrins/H2O2 System, 2013, 805806, 273-276.

63. M. N. M. Ibrahim, R. B. Sriprasanthi, S. Shamsudeen, F. Adam and S. A. Bhawani, Bioresources, A Concise Review of the Natural Existance, Synthesis, Properties, and Applications of Syringaldehyde, 2012, 7, 4377-4399.

64. Y. T. Zhu, J. Liu, Y. H. Liao, W. Lv, L. L. Ma and C. G. Wang, Topics in Current Chemistry, Degradation of Vanillin During Lignin Valorization Under Alkaline Oxidation, 2018, 376, 29.

65. E. M. Anderson, M. L. Stone, R. Katahira, M. Reed, G. T. Beckham and Y. Román-Leshkov, Joule, Flowthrough Reductive Catalytic Fractionation of Biomass, 2017, 1, 613-622.

66. C. X. Liu, Y. D. Yang, H. F. Gao, X. S. Bai and Z. J. Li, Korean Journal of Chemical Engineering, Preparation and enzymatic activity of Fe3O4-IDA-Ni/NAD kinase magnetic catalyst, 2020, 37, 475-481.

67. S. G. Santos, A. P. Marques, D. L. D. Lima, D. V. Evtuguin and V. I. Esteves, Industrial \& Engineering Chemistry Research, Kinetics of Eucalypt Lignosulfonate Oxidation to Aromatic Aldehydes by Oxygen in Alkaline Medium, 2011, 50, 291-298.

68. B. Beverskoga and I. Puigdomenech, $J$. Electrochem. Soc., Revised Pourbaix Diagrams for Copper at 25 to $300^{\circ} \mathrm{C}, 1997,14,3476-$ 3483.

69. E. A. Borges da Silva, M. Zabkova, J. D. Araújo, C. A. Cateto, M. F. Barreiro, M. N. Belgacem and A. E. Rodrigues, chemical engineering research and design, An integrated process to produce vanillin and lignin-based polyurethanes from Kraft lignin, 2009, 87, 1276-1292.

70. Z. Jiang, Z. Zhang, J. Song, Q. Meng, H. Zhou, Z. He and B. Han, ACS Sustainable Chemistry \& Engineering, Metal-Oxide-Catalyzed Efficient Conversion of Cellulose to Oxalic Acid in
Alkaline Solution under Low Oxygen Pressure, 2015, 4, 305-311.

71. Y. Yang, H. Gao, J. Feng, S. Zeng, L. Liu, L. Liu, B. Ren, T. Li, S. Zhang and X. Zhang, ChemSusChem, Aromatic Ester-Functionalized Ionic Liquid for Highly Efficient $\mathrm{CO} 2$ Electrochemical Reduction to Oxalic Acid, 2020.

72. L. Rocchetti, F. Veglio, B. Kopacek and F. Beolchini, Environ Sci Technol, Environmental impact assessment of hydrometallurgical processes for metal recovery from WEEE residues using a portable prototype plant, 2013, 47, 1581-8.

73. Z. Zhang and G. W. Huber, Chem Soc Rev, Catalytic oxidation of carbohydrates into organic acids and furan chemicals, 2018, 47, 13511390.

74. V. E. Tarabanko, N. A. Fomova, B. N. Kuznetsov, N. M. Ivanchenko and A. V. Kudryashev, Reaction Kinetics and Catalysis Letters, On the mechanism of vanillin formation in the catalytic oxidation of lignin with oxygen, 1995, 55, 161-170.

75. V. E. Tarabanko, D. V. Petukhov and G. E. Selyutin, Kinetics and Catalysis, New Mechanism for the Catalytic Oxidation of Lignin to Vanillin, 2004, 45, 569-577.

76. J. Liu, G. Chinga-Carrasco, F. Cheng, W. Y. Xu, S. Willfor, K. Syverud and C. L. Xu, Cellulose, Hemicellulose-reinforced nanocellulose hydrogels for wound healing application, 2016, 23, 3129-3143.

77. N. Ding, H. Liu, Y. Sun, X. Tang, T. Lei, F. Xu, X. Zeng and L. Lin, Journal of Cleaner Production, Lignin degradation in cooking with active oxygen and solid Alkali process: A mechanism study, 2021, 278, 123984.

78. A. Tamai, H. Goto, T. Akiyama and Y. Matsumoto, Holzforschung, Revisiting alkaline nitrobenzene oxidation: quantitative evaluation of biphenyl structures in cedar wood lignin (Cryptomeria japonica) by a modified nitrobenzene oxidation method, 2015, 69, 951- 
958.

79. Y. Zhao, Y. Zhao, R. Shi, B. Wang, G. I. N. Waterhouse, L. Z. Wu, C. H. Tung and T. Zhang, $A d v$ Mater, Tuning Oxygen Vacancies in Ultrathin $\mathrm{TiO} 2$ Nanosheets to Boost Photocatalytic Nitrogen Fixation up to $700 \mathrm{~nm}$, 2019, 31, e1806482.

80. J. Kim, W. Choi, J. W. Park, C. Kim, M. Kim and H. Song, J. Am. Chem. Soc., Branched Copper Oxide Nanoparticles Induce Highly Selective Ethylene Production by Electrochemical Carbon Dioxide Reduction, 2019, 141, 6986-6994.

81. Z. s. Hong, Y. Cao and J. f. Deng, Materials Letters, A convenient alcohothermal approach for low temperature synthesis of $\mathrm{CuO}$ nanoparticles, 2002, 52, 34-38.

82. S. C. Li, B. C. Hu, Y. W. Ding, H. W. Liang, C. Li, Z. Y. Yu, Z. Y. Wu, W. S. Chen and S. H. $\mathrm{Yu}$, Angew Chem Int Ed Engl, Wood-Derived Ultrathin Carbon Nanofiber Aerogels, 2018, 57, 7085-7090.

83. B. D. Lazic, S. D. Janjic, T. Rijavec and M. M. Kostic, Journal of the Serbian Chemical Society, Effect of chemical treatments on the chemical composition and properties of flax fibers, 2017, 82, 83-97.

84. J. Yang, M. Tu, C. Xia, B. Keller, Y. Huang and F. F. Sun, ACS Sustainable Chemistry \& Engineering, Effect of Fenton Pretreatment on $\mathrm{C} 1$ and C6 Oxidation of Cellulose and its Enzymatic Hydrolyzability, 2019, 7, 70717079 .

85. H. Lin, J. Strull, Y. Liu, Z. Karmiol, K. Plank, G. Miller, Z. Guo and L. Yang, Energy \& Environmental Science, High yield production of levulinic acid by catalytic partial oxidation of cellulose in aqueous media, 2012, 5, 9773.

86. M. M. Bashar, H. Zhu, S. Yamamoto and M. Mitsuishi, Cellulose, Highly carboxylated and crystalline cellulose nanocrystals from jute fiber by facile ammonium persulfate oxidation, 2019, 26, 3671-3684.

87. Y. Yang, X. Sun, G. Han, X. Liu, X. Zhang, Y.
Sun, M. Zhang, Z. Cao and Y. Sun, Angew Chem Int Ed Engl, Enhanced Electrocatalytic Hydrogen Oxidation on $\mathrm{Ni} / \mathrm{NiO} / \mathrm{C}$ Derived from a Nickel-Based Metal-Organic Framework, 2019, 58, 10644-10649.

88. Z. Y. Wu, P. Yin, H. X. Ju, Z. Q. Chen, C. Li, S C. Li, H. W. Liang, J. F. Zhu and S. H. Yu, Research (Wash $D C$ ), Natural Nanofibrous Cellulose-Derived Solid Acid Catalysts, 2019, 2019, 6262719 .

89. R. J. Moon, A. Martini, J. Nairn, J. Simonsen and J. Youngblood, Chem Soc Rev, Cellulose nanomaterials review: structure, properties and nanocomposites, 2011, 40, 3941-94.

90. F. Jiang, T. Li, Y. Li, Y. Zhang, A. Gong, J. Dai, E. Hitz, W. Luo and L. Hu, Adv Mater, WoodBased Nanotechnologies toward Sustainability, 2018, 30 .

91. B. Thomas, M. C. Raj, A. K. B, R. M. H, J. Joy, A. Moores, G. L. Drisko and C. Sanchez, Chemical Reviews, Nanocellulose, a Versatile Green Platform: From Biosources to Materials and Their Applications, 2018, 118, 1157511625.

92. J. Pennells, I. D. Godwin, N. Amiralian and D. J. Martin, Cellulose, Trends in the production of cellulose nanofibers from non-wood sources, 2019, 27, 575-593.

93. M. Thakur, A. Sharma, V. Ahlawat, M. Bhattacharya and S. Goswami, Materials Science for Energy Technologies, Process optimization for the production of cellulose nanocrystals from rice straw derived $\alpha$-cellulose, 2020, 3, 328-334.

94. S. Van den Bosch, T. Renders, S. Kennis, S. F. Koelewijn, G. Van den Bossche, T. Vangeel, A. Deneyer, D. Depuydt, C. M. Courtin, J. M. Thevelein, W. Schutyser and B. F. Sels, Green Chemistry, Integrating lignin valorization and bio-ethanol production: on the role of $\mathrm{Ni}-\mathrm{Al} 2 \mathrm{O} 3$ catalyst pellets during lignin-first fractionation, 2017, 19, 3313-3326.

95. J. K. Sun, H. L. Li, L. P. Xiao, X. Guo, Y. M. Fang, R. C. Sun and G. Y. Song, ACS 
Sustainable Chem. Eng., Fragmentation of Woody Lignocellulose into Primary Monolignols and Their Derivatives, 2019, 7, 4666-4674.

96. W. Wang, Q. Zhou, X. Fei, Y. He, P. Zhang, G. Zhang, L. Peng and W. Xie, CrystEngComm, Synthesis of $\mathrm{CuO}$ nano- and micro-structures and their Raman spectroscopic studies, 2010, 12, 2232.

97. Q. B. Zhang, K. L. Zhang, D. G. Xu, G. C. Yang, H. Huang, F. D. Nie, C. M. Liu and S. H. Yang, Progress in Materials Science, $\mathrm{CuO}$ nanostructures: Synthesis, characterization, growth mechanisms, fundamental properties, and applications, 2014, 60, 208-337.

98. Q. Song, F. Wang, J. Y. Cai, Y. H. Wang, J. J. Zhang, W. Q. $\mathrm{Yu}$ and J. Xu, Energy \& Environmental Science, Lignin depolymerization (LDP) in alcohol over nickel-based catalysts via a fragmentation-hydrogenolysis process, 2013, 6, 994-1007. 